\title{
DUSP6 mediates resistance to JAK2 inhibition and drives leukemic progression
}

Tim Kong ${ }^{1}$, Angelo Brunelli Albertoni Laranjeira ${ }^{1, \#}$, Kangning Yang ${ }^{2,3, \#, ~ D a n i e l ~ A . ~ C . ~ F i s h e r ~}{ }^{1}$, LaYow $\mathrm{Yu}^{1}$, Anthony Z Wang ${ }^{4}$, Marianna Ruzinova ${ }^{5}$, Jared S. Fowles ${ }^{1}$, Maggie J. Allen ${ }^{1}$, Hamza Celik $^{6}$, Grant A. Challen ${ }^{6}$, Sidong Huang ${ }^{2,3}$, and Stephen T. Oh ${ }^{1,7^{*}}$

${ }^{1}$ Division of Hematology, Department of Medicine, Washington University School of Medicine, St. Louis, MO, USA.

${ }^{2}$ Department of Biochemistry, McGill University, Montreal, Quebec, Canada.

${ }^{3}$ Goodman Cancer Research Centre, McGill University, Montreal, Quebec, Canada.

${ }^{4}$ Department of Neurological Surgery, Washington University School of Medicine, St Louis, MO, USA.

${ }^{5}$ Department of Pathology and Immunology, Washington University School of Medicine, St.

Louis, MO, USA.

${ }^{6}$ Division of Oncology, Department of Medicine, Washington University School of Medicine, St. Louis, MO, USA.

${ }^{7}$ Immunomonitoring Laboratory, Center for Human Immunology and Immunotherapy Programs, Washington University School of Medicine, St. Louis, MO, USA.

\#These authors contributed equally.

"Corresponding author: stoh@wustl.edu 


\begin{abstract}
Chronic myeloproliferative neoplasms (MPNs) exhibit a propensity for transformation to secondary acute myeloid leukemia (sAML), for which the underlying mechanisms remain poorly understood, resulting in limited treatment options and dismal clinical outcomes. Here, we performed bulk transcriptome profiling accompanied by single cell RNA-sequencing on CD34+ stem/progenitor cells from serial patient samples obtained at the chronic MPN and SAML phases, and identified aberrantly increased expression of dual-specificity phosphatase 6 (DUSP6) underlying disease transformation. Genetic and pharmacologic targeting of DUSP6 led to inhibition of S6 and JAK/STAT signaling, resulting in potent suppression of cell proliferation, while also reducing inflammatory cytokine production in primary samples. Furthermore, ectopic DUSP6 expression augmented proliferation and mediated JAK2 inhibitor resistance, while DUSP6 inhibition reduced colony-forming potential of JAK2 inhibitor-persistent patient cells. Mechanistically, DUSP6 perturbation dampened S6 signaling via inhibition of RSK1, which we identified as a second indispensable candidate associated with poor clinical outcome. Lastly, DUSP6 inhibition potently suppressed disease development across Jak2 V617F and MPL W515L MPN mouse models, and SAML patient-derived xenografts. These findings underscore DUSP6 in driving disease transformation and therapeutic resistance, and highlight the DUSP6-RSK1 axis as a novel, druggable pathway in myeloid malignancies.
\end{abstract}




\section{Introduction}

Myeloproliferative neoplasms (MPNs) are clonally derived from hematopoietic stem/progenitor cells (HSPCs) and typically harbor somatic mutations in one of three genes (JAK2, CALR, MPL) leading to aberrant activation of JAK-STAT signaling(1-3). Polycythemia vera (PV) and essential thrombocythemia (ET) are chronic MPNs characterized by overproduction of blood cells (erythrocytosis, thrombocytosis) and thrombohemorrhagic complications, while myelofibrosis (MF) is a more aggressive MPN subtype characterized by anemia, splenomegaly, bone marrow fibrosis, and constitutional symptoms (fever, night sweats, fatigue, weight loss)(4). Small molecule inhibitors of JAK2 provide symptomatic benefit for MPN patients, but they do not eradicate the underlying malignant clone, nor do they reliably prevent disease progression $(5,6)$. These findings suggest that other signaling pathways may be dysregulated, promoting survival of the malignant clone despite JAK2 inhibition.

Chronic MPNs exhibit a propensity for transformation to secondary acute myeloid leukemia (sAML), which imparts a dismal prognosis with a median survival of $<6$ months $(7)$. Leukemic transformation is often associated with the acquisition of additional mutations (e.g. TP53), and several studies have established that the presence of "high-risk" mutations (e.g. $A S X L 1, E Z H 2, S R S F 2, I D H 1 / 2)$ at the chronic MPN stage confers an increased risk of sAML transformation(7-10). However, the manner in which these genetic alterations contribute to leukemic transformation remains poorly understood. Despite vigorous research, therapies capable of reliably halting or preventing MPN disease progression remain elusive. Therefore, there is a pressing need to better define the mechanisms responsible for transformation of MPNs to sAML, which may translate to therapies capable of preventing and/or treating disease progression.

There has been emerging interest in the role and targeting of the MAPK/ERK signaling pathway in hematological malignancies $(11,12)$. DUSP6 is a member of the MAPK phosphatase family that is categorized into three subgroups based on sequence homology, subcellular 
localization, and substrate specificity(13-15). DUSP6 and other Class II DUSPs are highly selective for ERK while class I DUSPs (including DUSP1) act on JNK and p38 (in addition to ERK). Canonically, DUSP6 regulates signaling through ERK (and downstream signaling mediators) via a dynamic feedback regulatory axis: activation of MAPK signaling leads to induction of DUSP6 expression, followed by interaction of DUSP6 with ERK leading to dephosphorylation and inactivation of ERK(15). The specific role of DUSP6 is largely context dependent, such that aberrant DUSP6 expression may exert seemingly paradoxical effects on ERK and related signaling pathways, as illustrated by prior studies supporting an oncogenic role for DUSP6 in some cancers, but a tumor suppressor role in other settings $(14,15)$. Given these observations, DUSP6 may be an important mediator of disease burden and therapeutic response. Here, using multiomic approaches, we identify DUSP6 to be a critical driver of MPN disease progression, whose aberrant expression also confers resistance to JAK2 inhibition. We further exploit this dependency pharmacologically via DUSP6 targeting across in vitro, in vivo, and ex vivo MPN/sAML models. 


\section{Methods}

\section{Cell culture}

HEL (ATCC) and UKE-1 (Coriell Institute) cells were cultured in RPMI 1640 Medium ATCC modification and RPMI 1640 Medium (ThermoFisher, Waltham, MA), respectively, supplemented with $10 \%$ fetal bovine serum (FBS) and $1 \%$ penicillin/streptomycin. All cell lines were maintained at $37^{\circ} \mathrm{C}$ and $5 \% \mathrm{CO} 2$ and regularly tested for mycoplasma.

\section{Patient samples}

Patient and healthy donor control peripheral blood (PB) or bone marrow (BM) samples were obtained with written consent according to a protocol approved by the Washington University Human Studies Committee (WU no. 01-1014). Mononuclear cells (PBMC or BMMC) were obtained by Ficoll gradient extraction and cryopreserved according to standard procedures. Additional BMMCs were purchased from AllCells (Alameda, California). A list of patient samples utilized in this study is provided in Table 1.

\section{Compounds and antibodies}

Ruxolitinib, fedratinib, and trametinib were purchased from Selleck Chemicals (Houston, TX). BCI and BI-D1870 were purchased from MedChemExpress (Monmouth Junction, NJ). For in vivo treatment, $\mathrm{BCl}-\mathrm{HCl}$ and ruxolitinib were purchased from MedChemExpress. Human TPO was purchased from Peprotech (Rocky Hill, NJ). Protein Transport Inhibitor Cocktail (500X) was purchased from ThermoFisher.

Immunobloting antibodies were used as follows: DUSP6 (ab76310) from Abcam (Cambridge, MA). pRB S795 (9301), cleaved caspase-3 Asp175 (9661), pERK1/2 T202/7204 (9101), RSK1 (8408), p90RSK T359/S363 (9344), S6 (2317), pS6 S235/236 (4858), pSTAT3 Y705 (9131), 
pSTAT3 S727 (6E4), and pSTAT5 Y694 (9351) from Cell Signaling Technology (Danvers, MA). HSP90 (sc-13119), ERK1 (sc-93), and ERK2 (sc-154) from Santa Cruz Biotechnology (Dallas, TX). KLF2 (SAB1403063) from Sigma-Aldrich (St. Louis, MO).

Flow cytometry and cell sorting antibodies were used as follows and were from BD Biosciences (Franklin Lakes, NJ) : Alexa Fluor 488 Mouse Anti-Stat3 (pY705), PE-Cy7 Mouse anti-Stat5 (pY694), FITC Mouse Anti-Human CD34 (8G12), APC Mouse Anti-Human CD38 (HIT2), PE Mouse Anti-Human CD3 (UCHT1), PE Mouse Anti-Human CD19 (HIB19), PE Mouse Anti-Human CD14 (M5E2), PE Mouse Anti-Human CD16 (3G8), and PE Mouse Anti-Human CD7 (M-T701).

\section{Lentiviral transduction and constructs}

Lentiviral transduction with low multiplicity of infection (MOI) was performed using the protocol as described at http://www.broadinstitute.org/rnai/public/resources/protocols. Transduced cells were selected in puromycin or blasticidin for 2-4 days and plated for downstream assays immediately after selection.

Individual shRNA and ORF vectors used were from the Mission TRC library (Sigma), and ORF collections were developed by members of the ORFeome Collaboration (Sigma/TransOMIC), provided by the McGill Platform for Cellular Perturbation (MPCP) of Rosalind and Morris Goodman Cancer Research Centre at McGill University: control vector pLKO.5, shDUSP6 \#1 (TRCN0000233473), shDUSP6 \#2 (TRCN0000233471), shRPS6KA1 \#1 (TRCN0000001386), shRPS6KA1 \#2 (TRCN0000001388), shKLF2 \#1 (TRCN0000020727), $\quad$ shKLF2 \#2 (TRCN0000020728), control vector pLX304-eGFP, pLX304-DUSP6 (ccsbBroad304_00468), and pLX304-RPS6KA1 (ccsbBroad304_06896).

\section{Cell viability assays}


HEL, UKE-1, and patient PBMCs were seeded into 96-well plates at a density of $2 \times 10^{3}$ to $2 \times$ $10^{4}$ cells/well upon gene perturbation or treated with indicated doses of inhibitors and cultured for 72-96 hours. At endpoint, AlamarBlue viability reagent was added and absorbance was read in a BioTek microplate reader.

\section{Generation of persistent HEL cells}

HEL cells were cultured in the presence of $1 \mathrm{uM}$ of ruxolitinib or fedratinib for two months. Cells were pelleted and resuspended in $1 \mathrm{uM}$ of inhibitor weekly to maintain selection pressure.

\section{Immunoblotting}

$5 \times 10^{5}$ to $2 \times 10^{6}$ cells/well were seeded into 6 -well or 12 -well plates. After the indicated treatment duration, cells were washed with ice-cold PBS and lysed with protein sample buffer. Samples were processed with NuPAGE Novex Gel Electrophoresis Systems (ThermoFisher) followed by standard immunoblotting procedure.

\section{qRT-PCR}

RNA from plated cells were extracted with TRIzol (ThermoFisher). cDNA was synthesized using the Maxima First-Strand cDNA Synthesis Kit (ThermoFisher). Relative mRNA levels were measured through qRT-PCR using SYBR Green Master Mix (Roche) and normalized to the expression of $\beta$-actin (ACTB). Primer sequences are as follows:

ACTB forward, 5'-GTTGTCGACGACGAGCG-3'

ACTB reverse, 5'-GCACAGAGCCTCGCCTT-3'

DUSP6 forward, 5'-GTAGGGGTTGTGAATTGTGT-3'

DUSP6 reverse, 5'-ACCACCAATACCCACAACCA-3' 


\section{Flow cytometry, annexin $\mathrm{V}$ apoptosis assay, and cell cycle assay}

\section{Signaling flow cytometry}

$2.5 \times 10^{5} \mathrm{HEL}$ cells were seeded and treated with $1 \mu \mathrm{M} \mathrm{BCl}$ for 24 hours. Post treatment, cells were fixed with paraformaldehyde, washed with PBS, permeabilized with methanol, and stained with flow antibodies. Samples were run on BD FACSCanto II Cell Analyzer utilizing unstained controls, and downstream analysis was performed with FlowJo (Ashland, OR). Cell gating is as follows: SSC-A/FSC-A (size, live/dead), FITC-A/PE-Cy7-A (pSTAT3 Y705/pSTAT5 Y694).

\section{Annexin V apoptosis assay}

HEL cells were seeded at $4 \times 10^{5}$ cells/well and BCI, BI-D1870, or DMSO control were added for their indicated treatment duration. Protocol was followed per the PE Annexin V Apoptosis Detection Kit I (BD Biosciences). In brief, post treatment, cells were washed with PBS and resuspended in binding buffer, incubated with PE-Annexin $V$, and stained with 7-AAD viability staining solution. Cells were then analyzed by flow cytometry, in which AnnexinV+/7-AAD+ cells were identified as necroptotic, AnnexinV+/7-AAD- as apoptotic, and AnnexinV-/7-AAD- as healthy.

\section{$\underline{\text { Cell cycle assay }}$}

HEL cells were seeded at $4 \times 10^{5}$ cells/well and BCI, BI-D1870, or control were added for the indicated duration. Post treatment, cells were washed with PBS and fixed in $70 \%$ ethanol for 24 hours. Cells were washed with PBS and then treated with RNase, followed by staining with propidium iodide. Cells were then analyzed by flow cytometry.

\section{CD34+ cell patient microarray}

CD34+ cells from MF $(n=14)$ and sAML $(n=6)$ patient PBMCs and from normal BMMCs $(n=5)$ were sorted. Cryopreserved cells were thawed, filtered, and stained with the following 
immunostaining panel for sorting: CD34, CD38, CD3, CD19, CD14, and CD16 antibodies, in addition to 7-AAD viability marker. 7AAD- lin- CD34+ cells were sorted and mRNA was isolated, amplified, and converted to biotinylated sense-strand cDNA with WT Pico Reagent Kit (ThermoFisher). cDNA were then probed by GeneChipHuman Gene 2.0 ST Arrays (Affymatrix). Raw files were processed with the oligo package, quality controlled with AUC and RLE cut-offs, and normalized using the RMA-normalized log2-transformed algorithm. Differential gene expression analysis comparing sAML vs MF CD34+ samples was performed with Limma(16), where a Benjamini-Hochberg adjusted p-value cut-off of 0.05 was used.

\section{HEL RNA sequencing}

RNA from HEL parental, HEL-RuxR, Hel Fed-R, HEL 4h control, HEL 24h control, HEL 4h BCl, HEL 24h BCl, a second set of HEL 24h control, HEL 24h BI-D1870, and HEL 24h BCI + BI-D1870 were extracted using RNeasy Mini Kit (Qiagen) with DNase treatment in duplicate. Samples were then indexed, pooled, and sequenced by Illumina NovaSeq 6000 . Basecalls and demultiplexing were performed with Illumina's bcl2fastq software and a custom python demultiplexing program with a maximum of one mismatch in the indexing read. RNA-seq reads were then aligned to the Ensembl release 76 primary assembly with STAR version 2.5.1a(17). TMM normalization size factors in $\operatorname{EdgeR(18)}$ were calculated to adjust for samples for differences in library size. Ribosomal genes and genes not expressed in the smallest group size minus one samples greater than one count-per-million were excluded from further analysis. The TMM size factors and the matrix of counts were then imported into the R/Bioconductor package Limma(16). Differential expression analysis was then performed to analyze for differences between conditions and the results were filtered for only those genes with Benjamini-Hochberg false-discovery rate adjusted p-values less than or equal to 0.05 . Gene set enrichment analysis (GSEA) of MSigDb pathways was performed using the R/Bioconductor package GAGE(19) to test for changes in expression of the reported log 2 fold-changes reported by Limma in each term versus the background log 2 fold- 
changes of all genes found outside the respective term, and using GSEA(20) software with the Hallmark gene set, KEGG signaling and metabolism, or other curated gene sets to identify altered pathways.

\section{Single cell RNA-seq with surface TotalSeqA protein expression detection}

Cryopreserved BMMCs from two normal control donors (N34 and N39), and PBMCs from serial collections from three patients, including chronic MPN phase and subsequent sAML phase were utilized: Patient 381812 (MF post ET and SAML 12 months following), Patient 145790 (two collections from MF post ET separated by 30 months and SAML 25 months following), and Patient 374024 (chronic PV and sAML 6 months following). Non-lymphoid CD34 cells were identified by the following immunostaining panel: CD34, CD38, CD3, CD7, CD19, and 7AAD. 7AAD- CD3CD7- CD19- CD34+ cells were sorted and subsequently labeled with a panel of oligonucleotide conjugated antibodies (Table 9) according to TotalSeqA protocol from 10X Genomics (Pleasanton, CA). Oligonucleotide labeled cells were subjected to droplet bead capture, followed by cell lysis, reverse transcription, and amplification following protocol. Single cell derived cDNA libraries were sequenced on NovaSeq S4 cell sequencer (Illumina, San Diego, CA). Cellranger (10x Genomics, version 3.0.1) was used for transcript alignment, counting, and inter-library normalization of single cell samples. Seurat(21) (version 4.0.0) was used for cluster analysis. Cells containing fewer than 500 genes, a nCount value greater than the nCount value of the 93rd percentile of the entire sample, and more than $10 \%$ mitochondrial transcripts were removed, after which all filtered samples were merged. RNA reads were normalized with LogNormalize, ADT antibodies for surface protein expression were normalized with Centered Log-Ratio method, and data were scaled. Cell cycle phase was determined using CellCycleScoring, based upon on relative expression of phase-specific genes(22). Principle component analysis was performed on variable genes and the optimal number of PCs was selected to be 44 after JackStrawPlot analysis. Dimensionality reduction and visualization were performed with the uniform manifold 
approximation and projection (UMAP) algorithm and unsupervised graph-based clustering of cells was performed at a resolution of 0.8 . Differential gene expression analysis between populations/samples of interest was performed utilizing FindAllMarkers with Wilcoxon rank sum test. Gene set enrichment analysis of DEGs was run using fgsea(23) and the Hallmark gene set from msigdbr. Subsequent subsetting of samples was performed for downstream analyses as indicated. Trajectory analysis was performed with Monocle 2(24) (version 2.18.0) utilizing DEGs from each cell cluster. Dimensionality reduction was performed with reduceDimension method DDRTree and custom root state was set to HSC or earliest pluripotent progenitor cell population. DEG analysis was utilized to identify branch-specific genes at each branchpoint post-trajectory analysis. Pseudotime expression of genes of interest was performed across chronic MPN and sAML samples, and along disease states from trajectory analysis. Cell populations were identified using ADT antibody markers and gene set signatures provided in Supplementary Fig S4.

\section{CD34+ cell colony assay}

MPN and SAML patient PBMCs and normal BMMCs samples were thawed, filtered, and stained with CD34, CD38, CD3, CD19, CD14, and CD16 antibodies, in addition to 7-AAD viability marker. 7AAD- lin- CD34+ cells were sorted and seeded at 1,000 cells/mL in Methocult H4034 (StemCell) with the indicated inhibitors. Each condition was plated in duplicate or triplicate as indicated. Myeloid and erythroid colonies were counted after 10-14 days post-seeding.

\section{Mass cytometry (СуTOF)}

Signaling and cytokine CyTOF experiments utilizing HEL cells and patient PBMCs and BMMCs were conducted on a CyTOF2 mass cytometry (Fluidigm) with validated antibody panels and following protocols as previously described(25-29). In brief, primary cells were thawed, filtered, and counted on a hemocytometer. For primary sample signaling CyTOF, cells were then labelled with cisplatin, washed, and incubated with indicated stimulant or inhibitor for the indicated 
duration. Cells were then fixed with paraformaldehyde, permeabilized with perm buffer, and barcoded. Following, cells were stained with surface marker antibodies, permeabilized with methanol, stained with intracellular antibodies, and resuspended in DNA IR-intercalator. For primary cytokine CyTOF, cells were incubated with $\mathrm{BCl}$ and TPO for 4 hours, and protein transport inhibitor cocktail (eBioscience) was added at the 2 hour treatment mark. Following, cells were stained with surface markers antibodies, stained with cisplatin, and fixed with paraformaldehyde. Cells were then permeabilized with eBioscience perm buffer, stained with intracellular antibodies, fixed, barcoded, and suspended in Ir-intercalator. For persistent HEL cell signaling CyTOF, cells were labeled with cisplatin, treated with indicated inhibitors for 1 hour, fixed, barcoded, and permeabilized with methanol. Following, cells were stained with intracellular antibodies resuspended in Ir-intercalator. For sAML14 PDX CyTOF, the femurs of transplanted NSGS mice at end point were harvested and flushed, and bone marrow cells were collected and filtered. Cells were then labelled with cisplatin, washed, fixed, permeabilized, and barcoded. Following, cells were stained with surface marker antibodies, permeabilized with methanol, stained with intracellular antibodies, and resuspended in DNA IR-intercalator. Post CyTOF run, cell identities were debarcoded and data was analyzed in Cytobank (cytobank.org) and FlowJo (TreeStar, Eugene, OR). Lin- negative CD34+ cells were gated as follows: CD3-, CD19-, CD235-, CD71-, CD61-, CD14-, CD15-, CD16-, CD34+. For sAML PDX14, to separate human and mouse bone marrow cells, post CyTOF run cells were gated by surface expression of hCD45 and mCD45. CyTOF antibody panels are provided in Table 9.

\section{Immunofluorescence and immunohistochemistry}

Formalin fixed paraffin embedded (FFPE) blocks prepared from patient bone marrow core biopsies were sectioned to $5 \mu \mathrm{m}$ thickness on slides. Slides were deparaffinized by heating to $60^{\circ} \mathrm{C}$ for 2 hours, followed by serial exposure to xylene, $100 \%, 95 \%, 80 \%$, and $70 \%$ ethanol solutions, and water. Rehydrated slides were treated with $10 \mathrm{mM}$ Tris, $1 \mathrm{mM}$ EDTA, pH 9.0 for 30 
minutes at $96^{\circ} \mathrm{C}$ for antigen retrieval. Sections were blocked with $3 \%$ bovine serum albumin in phosphate buffered saline (PBS) for 30 minutes at room temperature. Sections were stained with mouse antibody to human DUSP6 antibody for $16-20 \mathrm{~h}$ at $4^{\circ} \mathrm{C}$, followed by washes with $0.5 \%$ Triton X-100 in PBS, PBS alone, and labeling with anti-mouse secondary antibody, Alexa 647 conjugate for $1 \mathrm{~h}$ at room temperature. Sections were counterstained with DAPI to visualize cell nuclei, and imaged on a Leica DM6 upright fluorescent microscope, using a Leica DFC 700T camera and LAS-X imaging suite.

For mouse histopathology, tibias were extracted and fixed in $10 \%$ neutral buffered formalin overnight at $4^{\circ} \mathrm{C}$. Post fixation, tibias were decalcified in $14 \%$ EDTA for 12 days and then rehydrated with serial exposure to $20 \%, 30 \%, 50 \%$, and $70 \%$ ethanol, all for 1 hour. Tibias were then rinsed in PBS and processed for paraffin embedding and sectioned at $5 \mu \mathrm{M}$. H\&E staining was performed by the Washington University Musculoskeletal Histology and Morphometry Core. Reticulin staining of bone marrow samples was performed on Ventana BenchMark Special Stains automated slide system (Ventana Medical Systems, Tucson, AZ, USA).

\section{Imaging mass cytometry}

Cryopreserved PBMCs from healthy control LRS2, MF20, and sAML1 were pelleted, fixed in 1.6\% formaldehyde for 10 minutes at room temperature followed by $10 \%$ neutral buffered formalin for 48h at $4^{\circ} \mathrm{C}$, and embedded in Histogel (ThermoFisher), followed by subsequent embedding in paraffin and sectioning, rehydration, antigen retrieval, and blocking as described for immunofluorescence. Antibodies for imaging mass cytometry were conjugated to metal-chelator complexes according to an established conjugation kit protocol (Fluidigm). Mass cytometry signals were recorded with the Hyperion imaging mass cytometry platform (Fluidigm). Exported mcd files were loaded into histoCAT++(30) and a median filter (size 3) was applied to all channels before image export. Antibodies panels are provided in Table 9. 


\section{In vivo mouse models}

All procedures were conducted in accordance with the Institutional Animal Care and Use Committee of Washington University.

\section{$\underline{\text { Normal mice }}$}

PBS vehicle or $\mathrm{BCl}-\mathrm{HCl}(25 \mathrm{mg} / \mathrm{kg}$ ) was administered intraperitonially once a day to 7 week-old C57BL/6 mice. Mice were treated for four weeks and peripheral blood was collected every Friday and cells were analyzed by Hemavet. Mice were sacrificed at endpoint, and body, spleen, and liver weights were recorded.

\section{$\underline{\text { Jak2 V617F model }}$}

Whole bone marrow was harvested from femurs and tibias of CD45.2 donor mice (6-12 weeks old) by flushing. Cells were resuspended and filtered in sort buffer (PBS containing $0.5 \%$ BSA and $2 \mathrm{mM}$ EDTA). 1 × $10^{5}$ bead-enriched Kit+ cells were collected from CD45.2 Jak2 V617F donor mice and transplanted via tail vein into CD45.1 lethally irradiated recipient mice. Recipient mice were irradiated with a total of 1,100 cGy, given as two separate doses of 550 cGy with 3-5 hours between doses. Within three hours of the last irradiation, mice were transplanted. For two weeks following transplant, the mice were caged with water containing $0.5 \mathrm{mg} / \mathrm{mL}$ Trimethoprim/sulfamethoxazole (Washington University Division of Comparative Medicine Pharmacy). Two weeks after transplant, peripheral blood was collected, and hematologic parameters were measured by Hemavet and flow cytometry. Mice were treated with PBS vehicle or $\mathrm{BCl}-\mathrm{HCl}(25 \mathrm{mg} / \mathrm{kg})$ intraperitonially daily. Treatment began two weeks after transplant, Monday through Friday. Mice were treated for four weeks and peripheral blood was collected every Friday and cells were analyzed by Hemavet and flow cytometry. Mice were sacrificed at endpoint, and body, spleen, and liver weights were recorded. 


\section{MPL W515L model}

Retroviral transduction was performed as described previously(31). Briefly, retrovirus was produced by transfecting $293 \mathrm{~T}$ cells with viral plasmids using jetPrime transfection reagent and viral supernatant was collected after 24 hours. Whole bone marrow was harvested from femurs and tibias of CD45.2 donor mice (6-12 weeks old) by flushing. The cells were resuspended and filtered in sort buffer (PBS containing 0.5\% BSA and 2mM EDTA). Isolated bead-enriched Kit+ cells were grown in 6-well plates for 2 days while undergoing spinoculation with the viral supernatant once per day by centrifugation at $350 \mathrm{xg}$ for 30 minutes. GFP expression was verified by flow cytometry prior to transplantation. $5 \times 10^{5}$ bead-enriched Kit+ cells retrovirally transduced with MPL W515L were transplanted via tail vein into CD45.1 lethally irradiated recipient mice (7 weeks old). Recipient mice were irradiated with a total of 1,100 cGy, given as two separate doses of 550 cGy with 3-5 hours between doses. Within three hours of the last irradiation, mice were transplanted. For two weeks following transplant, the mice were caged with water containing 0.5 $\mathrm{mg} / \mathrm{mL}$ Trimethoprim/sulfamethoxazole. Two weeks after transplant, peripheral blood was collected and hematologic parameters were measured by Hemavet and flow cytometry. Mice were treated with PBS vehicle or $\mathrm{BCl}-\mathrm{HCl}(25 \mathrm{mg} / \mathrm{kg})$ intraperitonially daily. Treatment began two weeks after transplant, Monday through Friday. Mice were treated for three weeks and peripheral blood was collected every Friday and cells were analyzed by Hemavet. Survival was monitored daily, and moribund mice were humanely sacrificed. Spleens were weighed at endpoint and normalized to mouse body weight at endpoint.

\section{$\underline{\text { sAML patient-derived xenograft (PDX) models }}$}

PDX models were performed following protocol as previously described $(32,33)$. Briefly, PBMCs

from sAML patients were isolated by Ficoll gradient extraction and CD34+ cells were isolated using magnetic enrichment (Miltenyi Biotec \#130-100-453) and cultured overnight in SFEMII 
media (StemCell Technologies \#09605) supplemented with Pen-Strep (50 Units/mL), human stem cell factor (SCF; $50 \mathrm{ng} / \mathrm{mL}$ ), human thrombopoietin (TPO; $50 \mathrm{ng} / \mathrm{mL}$ ), and human FLT3L (50 $\mathrm{ng} / \mathrm{mL}$ ). Human cells were transplanted into sublethally irradiated (200 cGy) 6-9 week-old NODscid-II2rg-null-3/GM/SF (NSGS; The Jackson Laboratory \#013062). NSGS mice were transplanted with $1 \times 10^{5}$ cells via intra-tibial injection under anaesthesia with intraperitoneal injection of ketamine/xylazine mixture (2 mg/mouse; KetaVed). Four-to-five weeks after transplant, peripheral blood was collected and engraftment was evaluated by flow cytometry. Mice were treated with PBS vehicle intraperitonially, $5 \%$ dimethyl acetamide $0.5 \%$ methylcellulose by oral gavage, daily intraperitoneal $\mathrm{BCl}-\mathrm{HCl}(25 \mathrm{mg} / \mathrm{kg})$, ruxolitinib $(90 \mathrm{mg} / \mathrm{Kg})$ by oral gavage twice a day, or combination. Treatment began four weeks after transplant for SAML11 and after five weeks for SAML14, Monday through Friday. Mice were treated for four weeks and peripheral blood was collected every Friday and cells were analyzed by flow cytometry. Spleens and livers were weighed at endpoint and normalized to mouse body weight at endpoint.

\section{Publicly available databases accessed}

Description and analyses of additional publicly available databases investigated in this study are as follows:

\section{OHSU BeatAML}

Clinical and expression data were obtained from Tyner et al.(34) and vizome.org. mRNA expression is indicated as normalized RPKM and samples compared were binned as NBM, priorMPN sAML, prior-MDS sAML, and de novo AML.

\section{GSE30029 De novo AML cohort(35)}

Expression arrays of CD34+ and CD34- sorted mononuclear cells from AML patients and normal CD34+ bone marrow cells. mRNA expression is indicated as quantile normalized value. 


\section{GSE53482 primary myelofibrosis cohort(36)}

Expression array of CD34+ from primary MF patients and CD34+ NBM. Gene expression value is indicated as log2 RMA signal. DEG analysis was performed by GEO2R comparing MF to NBM. For genes with multiple probes, the value with lowest adjusted $p$-value post-DEG was utilized.

\section{$\underline{\text { TCGA LAML(37) and other TCGA datasets }}$}

Gene expression and mutational data was accessed from cBioportal(38) and pearson correlations were performed. Clinical data were extracted from source publication. Kaplan-Meier survival data of RPS6KA1-stratified patients and log-rank p-value were calculated, where event free survival and overall survival of the patients with the highest $50 \%$ expression $(n=86)$ were compared to the bottom $50 \%(n=87)$.

For multivariate analysis, patient clinical information was retrieved from Supplemental Table 01 of TCGA LAML(37). RPS6KA1 expression was extracted from cBioportal from the TCGA LAML cohort. A modified table encompassing clinical information and RPS6KA1 expression was constructed for 173 patients and consisted of the following features: TCGA patient ID, RPS6KA1 expression, Expired? 3.31.12, Sex, Race, FAB, Age, \%BM blast, WBC, \%PB blast, RISK (Cyto), RISK (Molecular), 2017 ELN Genetic Risk, OS months 3.31.12, Chemotherapy (pooled into ATRA, induction, hypomethylating, or other), Transplant (pooled into none, auto, or other), mutations/alterations occurring in at least 5 patients and pooled as present or null: PML-RARA, MLL-partner, MYH11-CBFB, RUNX1-RUNX1T1, MLLT10-partner, BCR-ABL, GPR128-TFG, NUP98-NSD1, MLL-PTD, FLT3, NPM1, DNMT3A, IDH2, SRSF2, IDH1, RUNX1, TET2, TP53, NRAS, CEBPA, WT1, PTPN11, KIT, KRAS, MT-CO2, TTN, U2AF1, SMC1A, SMC3, STAG2, MTCYB, PHF6, ASXL1, FAM5C, FCGBP, MUC16, RAD51, \# of mutated/altered genes, and mutated recurrent genes. Univariate Cox proportional hazard models were used to assess the effect of 
each factor on overall survival. Only those variables significant in the univariate analysis were considered for multivariate Cox model. The final Cox model was fitted using a backward selection procedure. The diagnosis of model assumptions was performed using residual plots and utilized 170 observations.

RPS6KA1 expression data from the TCGA Pan-Cancer cohort was extracted from cBioportal. The number of patients $(n=)$ from each cancer subtype is as follows: Acute Myeloid Leukemia (173), Adrenocortical Carcinoma (78), Bladder Urothelial Carcinoma (407), Brain Lower Grade Glioma (514), Breast Invasive Carcinoma (1082), Cervical Squamous Cell Carcinoma (36), Cholangiocarcinoma (36), Colorectal Adenocarcinoma (592), Diffuse Large B-Cell Lymphoma (48), Esophageal Adenocarcinoma (181), Glioblastoma (160), Head and Neck Squamous Cell Carcinoma (515), Kidney Chromophobe (65), Kidney Renal Clear Cell Carcinoma (793), Liver Hepatocellular Carcinoma (366), Lung Adenocarcinoma (510), Lung Squamous Cell Carcinoma (484), Mesothelioma (87), Ovarian Serous Cystadenocarcinoma (300), Pancreatic Adenocarcinoma (177), Pheochromocytoma and Paraganglioma (178), Prostate Adenocarcinoma (493), Sarcoma (253), Skin Cutaneous Melanoma (443), Stomach Adenocarcinoma (412), Testicular Germ Cell Tumors (149), Thymoma (119), Thyroid Carcinoma (498), Uterine Carcinosarcoma (57), Uterine Corpus Endometrial Carcinoma (527), and Uveal Melanoma (80). RPS6KA1 expression is provided as RSEM (Batch normalized from Illumina HiSeq_RNASeq V2 (log2(value+1)).

\section{$\underline{\text { Tzelepis et al. CRISPR dropout screen(39) }}$}

Dropout candidates in AML cell lines (such as RPS6KA1) at FDR 10\% that were not essential in non-AML lines HT29 and HT1080 (\#non-AML = 0) were identified and plotted. 
RPS6KA1 gene dependency from DepMap(40) and expression data from the Cancer Cell Line

\section{Encyclopedia (CCLE)(41)}

Gene expression of DUSP family genes across AML cell lines, and RPS6KA1 (Expression Public 21Q1) were accessed from CCLE by the Broad Institute. Gene Effect scores (CERES) from CRISPR (Avana) Public 21Q1 were accessed from the DepMap portal by the Broad Institute.

$\underline{\text { Cell line drug sensitivity correlations(42) }}$

Fedratinib IC50 of AML cell lines were derived from Cancerxgene and the GDSC1 dataset, and correlated with CCLE RPS6KA1 mRNA expression. Area under curve (AUC) of apitolisib, KW-742-1, torin-2, WYE-125132, MK-2206, and PF-4708671 and DUSP6 expression were accessed from DepMap portal by the Broad Institute.

GSE153319 Serial scRNA-seq of CD34+ cells from primary MF patient(43)

Violin plots were constructed from individual barcoded cells across PMF, treatment PMF, and sAML timepoints, and relative fold change expression of $D U S P 6, K L F 2$, and $K L F 1$, in addition to associated statistical analyses were derived from source publication.

GSE125150 inducible KLF1-inducible iPSC macrophages(44)

p-values of gene expression comparisons of DUSP6 between untreated iKLF1.2 derived macrophages and tamoxifen-added iKLF1.2 derived macrophages were assessed.

GSE149119 murine bone marrow-derived macrophage Klf2-knockout(45)

Dusp6 expression between vehicle and KO were compared with Mann-Whitney U-test

GSE27602 murine KIf2-/- yolk sac erythroid cells(46) 
Multi-Chip Significance score (S-score) and expression comparison p-value of Dusp6 (probe 1415834_at) between WT and KIf2-/- samples were identified. As per source publication, probe with absolute S-score values greater or equal to 2.00 were considered to be significant.

\section{ChIP-seq}

Sequencing tracks were investigated using the Cistrome Data Browser and WashU Browser with the following accession numbers: Nfil3 (GSM1437733)(47); Atf3 (GSM2663858)(48), Fos (GSM1875490(49), GSM2663847(48)), Fos/2 (GSM1004808(50)), H3K27ac (GSM2974670(51), GSM851270(52)).

\section{Other statistical analysis}

Statistical analyses were performed using GraphPad Prism (San Diego, CA) and $R$ software. One-way ANOVA, two-way ANOVA, two-tailed Student's $t$ test, Mann Whitney $U$ test, and Pearson correlations were performed as indicated. ${ }^{*}, p<0.05 ;{ }^{* *}, p<0.01 ;{ }^{* * *}, p<0.001 ;{ }^{* * * *}, p$ $<0.0001$. All measurements were taken from distinct samples. All relevant assays were performed independently at least 3 times.

\section{Data availability}

Patient microarray, bulk RNA-sequencing, scRNA-seq data, and mass cytometry data will be made publicly available upon publication. Analyzed sequencing data are currently presented in Table 2, 3, 4, 5, 7, and 8 .

\section{Code availability}

$\mathrm{R}$ scripts utilized in this study are available from corresponding author upon request. 


\section{$\underline{\text { Results }}$}

Transcriptome analysis reveals enriched proliferative signaling pathways and elevated DUSP6 expression in secondary acute myeloid leukemia stem/progenitor cells

To understand alterations to the transcriptional landscape underlying MPN disease progression, we performed microarray analysis on sorted linage-negative (lin-) CD34+ HSPCs from $14 \mathrm{MF}$ and 6 sAML patients, in addition to sorted CD34+ bone marrow cells from 5 healthy donors. We compared MF and SAML patient samples and plotted the most differentially expressed genes (DEGs) (Supplementary Fig. S1A, Table 1). Hallmark gene set enrichment analysis (GSEA) of the top altered pathways revealed enhancement of numerous pathways including TNF- $\alpha$ signaling via NF $\kappa$ B, consistent with our previous findings $(25,27)$, in addition to interferon, IL6/JAK/STAT3, KRAS, IL2/STAT5, and PI3K/AKT/mTOR signaling in sAML vs MF HSPCs (Fig. 1A). Of the 457 statistically significant DEGs that were identified, notably, we observed enrichment of IL3RA, FLT3, and CSF1R (Fig. 1B) in SAML samples, which have been established as hyperactive disease drivers of de novo AML(53-55). These findings suggest shared cellular mechanisms underlying secondary and de novo AML. DUSP6, encoding a MAPK phosphatase that regulates ERK signaling, was also among the top DEGs and significantly elevated in SAML vs MF HSPCs (Fig. 1B, C), ranking higher than the only other DUSP family member identified, DUSP18, among the 23 DUSPs (Table 2).

Aberrant upregulation of DUSP6 expression in post-MPN sAML was corroborated by an independent database, the OHSU BeatAML cohort(34) (Fig. 1D), in which DUSP6 was also enriched in other AML subtypes such as prior-MDS sAML and de novo AML, further suggesting a conserved role in AML development from different origins. Additionally, we found increased DUSP6 in patient CD34+ AML cells compared to CD34+ NBM cells from a separate de novo AML cohort(35) (Supplementary Fig. S1B). Next, we confirmed elevated DUSP6 protein expression using immunofluorescence (IF) which revealed higher DUSP6 protein in bone marrow from sAML 
patients compared to MF and healthy donors (Fig. 1E, Supplementary Fig. S1C). Additionally, IF analysis of serial bone marrow from patient 381812 demonstrated a substantial increase in DUSP6+ cells at MF day 245 compared to day 0 (Fig. 1E). These findings were corroborated by imaging mass cytometry analysis revealing enrichment of CD34+ DUSP6+ cells from sAML1 compared to MF20 in addition to DUSP6 expression in subsets of monocytes, T cells, and B cells (Supplementary Fig. S1D). These data show that DUSP6 is upregulated in malignant blood cells, and during evolution of MPN to SAML.

\section{Single cell RNA sequencing of serial patient samples corroborates aberrant DUSP6 expression accompanying disease progression}

To understand patient-specific gene expression changes across disease evolution, we performed single cell RNA sequencing (scRNA-seq) in conjunction with TotalSeq surface protein marker detection on more than 50,000 sorted CD34+ cells of serial samples from three patients: patient 381812 at MF and SAML, patient 145790 at early MF, late MF, and SAML, and patient 374024 at PV and sAML- in addition to two NBM controls (N34, N39) (Fig. 2A). UMAP analysis showed tight clustering of cells from N34 and N39, whereas there was marked separation of MF and AML samples from 381812, and PV and SAML samples from 374024, suggesting distinct disease states (Fig. 2B). 145790 early MF clustered more closely to its late MF and AML stages, with the latter showing extensive overlap. Furthermore, there was proximal clustering between 381812 MF and 145790 early MF, and these MF stages were separated from 374024 PV. These observations support the notion that across patients there may be conserved similarities in HSPCs at an early MPN stage that branch into distinct entities during leukemic progression.

GSEA of the DEGs of the sAML stage to its paired chronic MPN showed TNF- $\alpha$ signaling via NFKB to be the top pathway enriched across all three patients (Supplementary Fig. S2A). Of the top 1,000 DEGs from each paired sample, 61 were shared across all 3 patients and 21 were in the same direction (all sAMLs showed upregulation or downregulation) (Fig. 2C, Table 3). 
Within this shared group of 21 aberrantly expressed genes, DUSP6 was elevated at the sAML stage from each of the three patients (Fig. 2D, Supplementary Fig. S2B). Notably, high DUSP6 expression was also evident at the late MF stage from 145790. This observation of a rise in DUSP6 expression late in the chronic MPN stage, but prior to overt leukemic progression is consistent with the notion of dysregulated DUSP6 expression as a driver, rather than simply a consequence, of disease progression.

Due to the intrinsic heterogeneity of sAML arising from antecedent PV vs MF, we further investigated the DEGs specifically between the two sAML evolved from MF (381812 and 145790), which shared 141 genes in the same direction (Supplementary Fig. S3A, Table 3). In addition to DUSP6, differential expression of KLF transcription factor family members was identified, notably the decreased expression of $K L F 1$ and increase of $K L F 2$, which have been shown to play important roles in progenitor cell differentiation(56). Other transcription factors enriched included NFIL3 (Nuclear factor, interleukin 3 regulated) in addition to its interacting partners including ATF3, FOS, and FOSL2 that complex to form AP-1 and/or other multi-unit transcriptional regulators (Supplementary Fig. S3B). Publicly available ChIP-seq tracks(47-52) showed binding of these transcription factors to the Dusp6 locus which also overlap with H3K27ac activation marks (Supplementary Fig. S3C), suggesting potential regulation of Dusp6 by these canonical transcription factors. The family of early growth response (EGR) transcription factors was also enriched and has been described to demonstrate interplay with MAPK pathways(57). To substantiate these findings, we examined co-expression between DUSP6 and these enriched genes of interest across our patient microarray and four additional publicly available patient cohorts(34-37). Overall, a consistent correlation of these genes with DUSP6 was identified, with a negative correlation with KLF1 and a significant positive correlation observed with $K L F 2$ that was significant across all five cohorts (Supplementary Fig. S3D). Supporting this, correlation analysis of single cells from 381812 showed $K L F 2$ to be the most positively correlated gene with DUSP6, while KLF1 was among the 10 most negatively correlated genes (Supplementary Fig. 
S3E). These findings identify specific transcriptional programs as potential regulators of aberrant DUSP6 expression across disease progression.

We then explored the cellular and transcriptional landscapes of MF and SAML stages in 381812 by first delignating major cell populations (Fig. 2E, Supplementary Fig. S4A, S4B). 381812 MF exhibited predominant enrichment of pluripotent HSCs, along with skewing of erythroid lineage populations, while 381812 sAML was largely comprised of more committed myeloid progenitors. We investigated if the elevated DUSP6 expression at the SAML stage was indicative of high DUSP6 in a distinct SAML subpopulation and/or if it was consistently elevated across all sAML clusters. We observed the latter, in sAML clusters having elevated DUSP6 relative to MF clusters, and moreover, there was a similar pattern of enrichment of KLF2 and suppression of KLF1 among the dominant sAML clusters (Fig. 2F). Moreover, trajectory-based differential expression analysis revealed bifurcation of the MF and SAML samples, which overlapped with erythroid/megakaryocyte and myeloid identities, respectively, and identified DUSP6, KLF2, and KLF1 among the significant branch point determinants (Fig. 2G, Table 4). Differentiation trajectory pseudotime analysis revealed concomitant elevation of DUSP6 and $K L F 2$ and suppression of $K L F 1$ across state trajectories and disease progression from MF to sAML in 381812, which was further highlighted by similar trajectories in 374024 from PV to sAML and 145790 from early MF to late MF to sAML (Fig. 2H, Supplementary Fig. S5). In addition, we corroborated our findings in a separate serial CD34+ scRNA-seq dataset(43) of a PMF patient at different timepoints along transformation to SAML, in which we observed elevation of DUSP6 and $K L F 2$, in concomitant with decreased KLF1 through chronic MPN to sAML progression (Supplementary Fig. S6A-C).

Further in support of our scRNA-seq findings, we also observed decreased KLF1 expression and increased KLF2 expression in sAML vs MF in our CD34+ patient microarray (Supplementary Fig. S6D). We sought to validate the functional effect of $K L F 2$ in the regulation of DUSP6, where its knockdown by shRNA in JAK2 V617F mutant HEL cells led to 
downregulation of DUSP6 mRNA and protein (Supplementary Fig. S6E S6F), and significantly inhibited proliferation (Supplementary Fig. S6G). Finally, publicly available databases revealed decreased DUSP6 expression upon induction of KLF1 in iPSC-derived macrophages(44), while Klf2-knockout in mouse bone marrow derived macrophages(45) and embryonic yolk sac erythroid cells(46) showed decreased Dusp6 (Supplementary Fig. S6H, S6J). Altogether, bulk and singlecell sequencing analyses on primary patient samples demonstrate that DUSP6 elevation is a marker of disease progression from MF to SAML, which may be regulated by a host of interplaying transcription factors including $K L F 1$ and $K L F 2$.

\section{DUSP6 inhibition suppresses JAK-STAT and S6 signaling pathways}

Given the potential role of DUSP6 in driving disease progression, we sought to further delineate its function in MPN/AML. From the Cancer Cell Line Encyclopedia (CCLE)(41), we first assessed the mRNA expression among DUSP family genes across 35 AML cell lines and observed high expression of DUSP6, which was also elevated in HEL cells (Supplementary Fig. S7A, S7B). Pharmacologic inhibition of DUSP6 via BCI led to a dose-dependent decrease in HEL cell viability (Supplementary Fig. S7C). At low concentrations, 24 hour BCI treatment increased ERK phosphorylation in HEL cells, consistent with the inhibitor's capacity to suppress phosphatase activity (Supplementary Fig. S7D). Somewhat unexpectedly, higher BCl concentrations abolished ERK phosphorylation in conjunction with induction of apoptosis through increased cleavage of caspase 3. To determine if apoptotic and cell cycle inhibition profiles were a consequence of diminished ERK signaling, we treated HEL cells with the MEK1/2 inhibitor trametinib. Sub-micromolar doses of trametinib were sufficient to completely eradicate ERK phosphorylation but did not significantly alter cell viability or induce apoptosis (Supplementary Fig. S7C, S7D), suggesting that DUSP6 may play additional roles in signal transduction beyond direct ERK modulation to drive cancer survival. 
To uncover novel effectors of DUSP6, we probed other predominant cancer signaling pathways and observed dose-dependent suppression of pS6 in addition to pSTAT3 and pSTAT5 in HEL cells by $\mathrm{BCl}$ treatment that was not observed with trametinib (Fig. 3A, Supplementary Fig. S7E, S7F). Further cell cycle and viability profiling showed $\mathrm{BCl}$ led to suppression of RB phosphorylation, induced G1 arrest, and induced apoptosis through increased annexin V staining (Fig. 3A-C). We also performed RNA-seq on HEL cells treated with 4 hours and 24 hours $\mathrm{BCl}$, in which top downregulated GSEA pathways included E2F targets and G2M checkpoint (Fig. 3D, Supplementary Fig. S7G, Table 5), which were more pronounced with longer duration treatment. In addition to cell cycle related pathways, downregulation of MTORC1 signaling pathway was observed, consistent with signaling suppression in immunoblots. To confirm that the effects of $\mathrm{BCl}$ were DUSP6-dependent, genetic approaches were utilized to perturb DUSP6 expression. Indeed, knockdown of DUSP6 with independent shRNAs also led to suppression of pS6 and pSTAT3 in conjunction with reduced HEL viability (Fig. 3E, 3F). In contrast, ectopic expression of DUSP6 increased cell viability, further suggesting an oncogenic role of DUSP6 (Supplementary Fig. S7H, S7I). DUSP6 overexpression also conferred greater resistance to $\mathrm{BCl}$ treatment compared to control vector (Supplementary Fig. S7J), further validating DUSP6 targeting via BCI.

Consistent with the increased DUSP6 expression upon disease progression in patient samples, sAML peripheral blood mononuclear cells (PBMCs) were also more sensitive to $\mathrm{BCl}$ compared to MPN PBMCs and normal bone marrow mononuclear cells (BMMCs) (Fig. 3G), and BCl-treatment led to higher cleaved caspases 3 levels in CD34+ sAML cells compared to NBM (Fig. $3 \mathrm{H}$ ). In lin- CD34+ colony formation assays, $\mathrm{BCl}$ significantly reduced the colony forming units from sAML samples, with smaller MF and sAML colonies observed compared to NBM, (Fig. 3I, Supplementary Fig. S7K). To better investigate BCl's potency on distinct cell populations from primary samples and improve signal resolution to single cells, we performed multiplex profiling using suspension mass cytometry (CyTOF). Surface marker-guided dimensional clustering of sAML4 showed a large population of lin- CD34+ HSPCs, which demonstrated strong induction of 
pSTAT1, pSTAT3, pSTAT5, and pS6 upon treatment with JAK-STAT pathway activator thrombopoietin (TPO; Supplementary Fig. S8A, S8B), which was diminished by BCl co-treatment. Similar findings were observed in additional MF and sAML patient samples (Fig. 3J). Further mass cytometry analysis identified CD14+ monocytes as the predominant cytokine producing population, consistent with our previous studies(25) (Supplementary Fig. S8C). Upon TPO stimulation, IL-8 and in particular MIP-1ß/CCL4 were strongly upregulated in CD14+ monocytes but were nearly completely abrogated with addition of $\mathrm{BCl}$ (Supplementary Fig. S8D). TPOinduced hyperproduction of MIP-1ß/CCL4 in CD16+ monocytes and CD123+ cells from sAML5 were also suppressed by $\mathrm{BCl}$ (Supplementary Fig. S8E). Collectively, these novel findings demonstrate that DUSP6 drives tumorigenicity in MPNs/sAML, and DUSP6 inhibition suppresses dominant pS6/STAT signaling pathways and suppresses inflammatory cytokine production.

\section{DUSP6 regulates S6 phosphorylation in MF and AML via indispensable RSK1}

Alterations in PI3K/AKT/mTOR signaling pathways identified from bulk transcriptome and scRNA-seq analyses, in conjunction with observed suppression of pS6 after DUSP6 inhibition, led us to further explore downstream signaling mechanisms. Investigation of canonical regulators of S6 activity such as PI3K/AKT/mTOR and ribosomal S6 kinase (S6K) revealed a strong correlation between DUSP6 and the area under curve (AUC) of inhibitors against these targets across multiple AML cell lines (Supplementary Fig. S9A). We hypothesized that these signaling nodes may be crucial to AML and subsequently explored a publicly available CRISPR dropout screen(39) which identified genes indispensable for survival of AML cell lines (MOLM-13, MV411, HL-60, OCI-AML2, and OCl-AML3) but not for non-AML control cell lines (HT29 and HT1080). RPS6KA1 (also known as ribosomal protein S6 kinase alpha-1, RSK1 or p90S6K) was among the 27 targets whose inhibition was lethal to at least 4 out of 5 AML lines, and was dispensable in both control lines (Supplementary Fig. S9B). RSK1 is the p90 subunit which together with beta-subunit p70S6K (RPS6KB1) forms S6K to phosphorylate S6. Consistent with 
these findings, we observed that RPS6KA1 expression was highest in AML across a large collection of cancer cell lines from the Cancer Dependency Map Project (DepMap)(40) (Fig. 4A), and that AML was most sensitive to CRISPR-Cas9 mediated knockout of RPS6KA1 compared to other cancers (where a lower CERES score is indicative of an essential gene, a score of 0 indicates non-essential, and a score of -1 is comparable to the median score of pan-essential genes; Figure 4B). Elevated expression of RPS6KA1 was also observed in the TCGA Pan-Cancer cohort (10,071 total patients), in which patients with AML had the second highest expression out of 31 distinct cancer subtypes (Supplementary Fig. S9C).

In keeping with the dependency of RSK1 in AML cells, Kaplan-Meier event free survival and overall survival analysis of AML patients from TCGA showed significantly worse outcome for AML patients with higher RPS6KA1 expression (Fig. 4C, 4D). Multivariate analysis using Cox proportional-hazards model on overall survival showed RPS6KA1 expression to be significant $(\mathrm{HR}=1.60,95 \% \mathrm{Cl}: 1.10,2.34)$ and comparable to other important factors: age $(\mathrm{HR}=1.04,95 \%$ Cl: $1.02,1.05), W B C(H R=1.01,95 \% \mathrm{Cl}: 1.00,1.01)$, molecular risk classification of poor $(H R=$ 2.52, 95\% Cl: $1.29,4.94)$, alterations in TP53 (HR = 2.99, 95\% Cl: 1.49. 6.00), RUNX1 (HR = 1.99, 95\% Cl: 1.10, 3.60), and FLT3 (HR = 1.92. 95\% Cl: 1.23, 2.99; Table 6). These findings demonstrate that aberrant RPS6KA1 expression is associated with poor prognosis in AML and indicate that RSK1 represents a candidate target for therapeutic intervention.

Treatment of HEL and a second JAK2-mutant leukemia line UKE-1 with RSK1/2/3/4 inhibitor BI-D1870 led to a dose-dependent decrease in cell viability concurrent with reduced phosphorylation of pRSK1 at key activation residues T359/S363 and the suppression of pS6 (Fig. 4E, 4F). Importantly, RPS6KA1 knockdown phenocopied signal suppression seen with pan-RSK inhibition and significantly reduced HEL viability (Supplementary Fig. S9D, S9E), consistent with the AML CRISPR-KO phenotype (Fig. 4B). BI-D1870 treatment also inhibited cell cycle progression by arresting cells in G2 phase and led to increased cellular apoptosis (Fig. 4F-H). In addition, RNA-seq analysis of HEL cells treated with BI-D1870 for 24 hours or DMSO control 
revealed downregulation of PI3K-AKT signaling among the top altered GSEA KEGG signaling and metabolism pathways upon RSK1 inhibition (Fig. 4I, Table 7). Suppression of mediators of G2/M transition including WEE1, PLK1, CDK1, and CCNA2 (Table 7) was also observed in BID1870 treated cells, and the top 50 DEGs in the PI3K-AKT signaling pathway showed suppression of other cell cycle G1/S phase mediators CCND1 and CDK6 and upregulation of CDKN1A (Fig. 4J). In accordance with our annexin $\mathrm{V}$ assay, downregulation of apoptosis regulators $B C L 2$, and upregulation of $B C L 2 L 11$ and smaller isoform $M C L 1$ were also observed upon BI-D1870 treatment. Mass cytometry of MF patient samples treated with BI-D1870 showed inhibition of TPO-induced pS6 and p4E-BP1 signaling in lin- CD34+ HSPCs, but minimal effect on phosphorylation of STAT proteins, suggesting BI-D1870 largely targets downstream of mTOR (Fig. 4K, Supplementary Fig. S9F). BI-D1870 also preferentially suppressed formation of myeloid colonies from MF and sAML CD34+ HSPCs compared to normal bone marrow (Fig. 4L). These findings establish RPS6KA1 as a novel biomarker predicting patient prognosis and an essential candidate in MPN/AML to be exploited therapeutically.

We observed similar elevated expression of RPS6KA1 at single cell resolution in CD34+ cells from 381812 sAML compared to MF (Supplementary Fig. S9G), and in bulk CD34+ sAML patient cells, where a strong correlation with DUSP6 was also noted (Fig. 4M, Supplementary Fig. $\mathrm{S} 9 \mathrm{H})$. Given this observed relationship, we sought to determine if suppression of pS6 after DUSP6 perturbation was mediated through RSK1 inhibition. $\mathrm{BCl}$ treatment in $\mathrm{HEL}$ and UKE-1 cells led to reduced T359/S363 phosphorylation of RSK1, which was consistently suppressed in DUSP6 knockdown cells (Fig. 4N, 4O). In contrast, RPS6KA1 overexpression conferred resistance to $\mathrm{BCl}$ (Supplementary Fig. S9I). Therefore, we explored combination therapy with the addition of $\mathrm{BCl}$ to sub-maximal doses of BI-D1870, which suppressed HEL and UKE-1 viability more than each agent alone (Supplementary Fig. S9J). GSEA of RNA-seq of HEL cells treated with both $\mathrm{BCl}$ and $\mathrm{BI}-\mathrm{D} 1870$ showed amalgamation of altered pathways observed in monotherapy, demonstrating suppression of JAK/STAT and MTORC1 signaling pathways, E2F 
targets, and increased p53 pathway compared to DMSO control (Supplementary Fig. S9K). Mass cytometry analysis of lin- CD34+ cells from primary sample MF103 revealed suppression of pS6, pSTAT1/3/5, and p4E-BP1 proteins with $\mathrm{BCl}$ and BI-D1870 treatment, demonstrating combined signal-suppressive phenotypes from each inhibitor (Supplementary Fig. S9I). Taken together, these studies characterize a novel DUSP6-RSK1-S6 axis that is prognostic of patient outcome and of which perturbations in this cascade potently suppress MPN/sAML.

\section{DUSP6 inhibition overcomes persistence to fedratinib in vitro and ex vivo}

Prolonged suppression of driver pathways in cancer through single agent therapy inevitably leads to resistance and selection of drug-persistent clones, thus requiring further understanding of these dynamic alterations to amend therapeutic strategies. Cancer persistence is observed in the clinic, as JAK2-targeted therapies fail to eradicate the underlying MPN malignant clone, rendering MPN patients susceptible to leukemic transformation. We sought to mimic JAK2 inhibitor persistence in vitro via prolonged culturing of HEL cells in the presence of either ruxolitinib or fedratinib to generate ruxolitinib-persistent (Rux-P) and fedratinib-persistent (Fed-P) cells lines (Fig. 5A). RNA-seq analysis revealed upregulation of DUSP6 in Rux-P cells and Fed-P cells compared to parental HEL cells (Fig. 5B, Table 8). We also observed a relative increase of expression in a curated gene set of "DUSP6 targeted genes upregulated"(58) in FedP cells (Fig. 5C). Ectopic DUSP6 expression mediated resistance to both fedratinib and ruxolitinib in parental HEL cells (Fig. 5D), and upon JAK2 inhibition these cells retained pS6 but not pSTAT3, implicating pS6 as a key effector mediating resistance to JAK2 inhibitors (Fig. 5E). Notably, DUSP6 overexpression rescued pSTAT3 despite $\mathrm{BCI}$ treatment, suggesting indirect modulation of STAT signaling via BCI. In contrast, knockdown of DUSP6 further sensitized parental HEL cells to fedratinib and ruxolitinib (Fig. 5F, 5G). We also observed a trend of elevated DUSP6 expression correlating with decreased sensitivity to fedratinib in 18 AML cell lines (Supplementary Fig. S10A). 
These findings highlight a pivotal role for DUSP6 in mediating resistance to JAK2 inhibitors in AML.

Notably, Fed-P HEL cells showed similar sensitivity to $\mathrm{BCl}$ compared to the parental line (Supplementary Fig. S10B), while the addition of fedratinib to $\mathrm{BCl}$ also further suppressed cell viability in HEL parental cells (Supplementary Fig. S10C). Mass cytometry analysis of intracellular signaling changes in Fed-P cells revealed elevated phosphorylation of STAT1/3/5, AKT, and S6, relative to the parental line (Fig. $5 \mathrm{H}$ ). $\mathrm{BCl}$ or fedratinib alone suppressed signaling through these pathways with combination treatment demonstrating enhanced inhibition, particularly of pS6 (Fig. 5I). Taken together, these findings demonstrate that $\mathrm{BCl}$ alone or in combination with fedratinib can effectively inhibit cell proliferation through suppression of JAK-STAT and S6 signaling in both naïve and fedratinib-persistent cells.

To extend these findings ex vivo, we examined serial samples from MF post-ET patient 973039 obtained prior to initiation of JAK2 inhibitor treatment (T1) (Fig. 5J). Upon poor clinical response to ruxolitinib, patient 973039 was switched to fedratinib treatment for 18 months with subsequent development of worsening anemia, a hallmark of disease progression and poor response to therapy, at which timepoint an additional sample was collected (T2). Consistent with disease progression, targeted sequencing revealed acquisition of TET2, EZH2, and SUZ12 mutations, in addition to a second $A S X L 1$ mutation, at T2. We then isolated the CD34+ population and performed colony assays. 973039 T2 showed relatively decreased erythroid colonies and increased myeloid colonies compared to 973039 T1 and normal bone marrow (Fig. 5K), consistent with the clinical observation of worsening anemia. CD34+ cells from the later T2 timepoint were less susceptible than normal or T1 cells to inhibition of colony formation in the presence of fedratinib. Fedratinib also preferentially reduced erythroid colonies in all three samples, whereas $\mathrm{BCl}$ treatment reduced overall colony formation to a similar degree. Notably, the addition of $\mathrm{BCl}$ to fedratinib suppressed the residual, persistent colonies from the fedratinibalone treatment in 973039 T2. Paired mass cytometry analysis of 973039 T2 showed that $\mathrm{BCl}$ 
was sufficient to reverse TPO induction of pSTAT1, pSTAT3, pSTAT5, and pS6 (Fig. 5I). In sum, these data indicate that DUSP6 targeting via $\mathrm{BCl}$, either alone or in combination with fedratinib, represents a potentially effective and novel therapeutic avenue to overcome both naïve and JAK2 inhibitor-persistent MPNs.

\section{DUSP6 inhibition suppresses disease burden and progression across multiple MPN and}

\section{sAML mouse models}

We then assessed the functional impact of DUSP6 targeting in vivo across MPN and SAML murine models. We first utilized Jak2 V617F knock-in mice, which exhibit features of PV including erythrocytosis, leukocytosis, and splenomegaly(59). Mice transplanted with Jak2 V617F-mutant cells were treated with vehicle or $25 \mathrm{mg} / \mathrm{kg} \mathrm{BCl} 5$ days on, 2 days off (Fig. 6A). Hematocrit remained abnormally elevated in vehicle-treated control mice but was significantly reduced within the first week of $\mathrm{BCl}$ administration and sustained across the entire treatment duration (Fig. 6B). White blood cell (WBC) counts, individual WBC differentials, and platelet counts were similarly significantly suppressed in BCl-treated mice compared to vehicle-treated controls (Fig. 6B, Supplementary Fig. S11A). There was no significant difference in body weight in treatment groups, whereas $\mathrm{BCI}$ reduced splenomegaly and corrected liver weights (Fig. 6B, Supplementary Fig. S11B). Importantly, BCl treatment of primary wildtype mice did not pathologically cause anemia or other cytopenias, or decrease spleen and liver weights (Supplementary Fig. S11C), suggesting potency and specificity of $\mathrm{BCl}$ against MPN disease compared to normal tissue and confirming $\mathrm{BCl}$ does not exert non-specific myelosuppressive effects.

We next evaluated the efficacy of $\mathrm{BCl}$ in the MPL W515L model, which confers a robust MF phenotype with marked leukocytosis, bone marrow fibrosis, and early lethality(60). Kit+ cells from CD45.2 mice were transduced with MPL W515L retrovirus and transplanted into lethallyirradiated CD45.1 recipient mice (Fig. 6C, Supplementary Fig. S11D). Mice receiving 25 mg/kg $\mathrm{BCl}$ showed no difference in hematocrit compared to vehicle but had significant reduction of 
leukocytosis and near significant reduction of normalized spleen weight (Fig. 6D, Supplementary Fig. S11E). Pathological thrombocytosis was also significantly reduced by BCI (Fig. 6D). While vehicle-treated mice were all moribund by day $30, \mathrm{BCl}$-treated mice exhibited markedly prolonged survival (Fig. 6E). Bone marrow H\&E staining at endpoint demonstrated evident suppression of fibrosis and megakaryocyte hyperplasia in BCl-treated mice (Fig. 6F). Complementary immunohistochemical staining ( $\mathrm{IHC}$ ) showed reduction of reticulin with $\mathrm{BCl}$ treatment (Fig. 6F). Taken together, these findings demonstrate that $\mathrm{BCl}$ is efficacious in reducing disease burden of both PV and MF MPN mouse models.

To further recapitulate human disease, we adopted a novel, patient-derived xenograft (PDX) humanized animal system in which engrafted cells propagate MPN features(32,33) (Fig. 7A). CD34+ cells from sAML post-MF 718407 (sAML11) were transplanted into NSGS mice, and engrafted mice were treated with $25 \mathrm{mg} / \mathrm{kg} \mathrm{BCl}$ or vehicle. $\mathrm{BCl}$ treated mice showed marked reduction in percentage of human CD45 (hCD45) cells in both peripheral blood and bone marrow (Fig. 7B). There was reversal of splenomegaly but no changes to liver weights at end point (Fig. 7B)

We utilized a second PDX model engrafted with CD34+ cells from post-ET/MF sAML postMF 784981 (sAML14) to explore efficacy of combination therapy with ruxolitinib and BCl. Similar to PDX sAML11, potent suppression of $\mathrm{PB}$ hCD45 engraftment was observed with $\mathrm{BCl}$ monotherapy compared to vehicle (Fig. 7C). Ruxolitinib treatment also reduced PB hCD45 engraftment, with combination treatment $(\mathrm{BCl}+$ ruxolitinib) further suppressing $\mathrm{PB}$ hCD45 engraftment compared to ruxolitinib alone. $\mathrm{BCl}$, ruxolitinib, and combination treatment also all significantly suppressed BM hCD45 engraftment compared to vehicle and showed a trend in reduction of spleen but not liver weight (Fig. 7C, Supplementary Fig. S11F). To assess the degree of signaling suppression across the four treatment conditions, we performed mass cytometry on flushed bone marrow cells from sAML14 PDX mice (Supplementary Fig. S11G). BM hCD45+ cells isolated from $\mathrm{BCl}$, ruxolitinib, and $\mathrm{BCl}+$ ruxolitinib treatment groups showed relative inhibition of 
pSTAT1/3/5, pS6, and Ki67 compared to vehicle, in conjunction with increased cleaved caspase 3 (Fig. 7C). Mouse BM hCD45+ cells (mCD45+), also showed suppression of STAT phosphorylation but no obvious trend in pS6, Ki67, or cleaved caspase 3 . These experiments functionally validate signal suppression in vivo and demonstrate selectivity of $\mathrm{BCl}$ and ruxolitinib alone or in combination against engrafted malignant cells compared with normal tissue. Thus, our findings demonstrate a consistent reduction in disease burden across humanized and MPN mouse models, and highlight DUSP6 inhibition as a promising therapeutic approach. 


\section{Discussion}

Using bulk expression profiling and single-cell sequencing approaches on serial MPN and sAML patient samples, we identify aberrant overexpression of DUSP6 and downstream effector RPS6KA1 driving treatment resistance and disease progression. We target these dependencies using small molecule inhibitors $\mathrm{BCl}$ and $\mathrm{BI}-\mathrm{D} 1870$, and through mass cytometry multiplex profiling highlight suppression of dominant STAT and S6 proliferation pathways across cell lines and primary samples. Finally, we demonstrate selective efficacy of $\mathrm{BCl}$ across in vivo models of $\mathrm{PV}$ and $\mathrm{MF}$, and show that $\mathrm{BCl}$ treatment reduces disease burden alone and potentiates ruxolitinib efficacy in SAML PDX models.

Clinical observations of JAK2 inhibitors alone failing to eradicate the underlying malignant clone and limited anti-proliferative effects of MEK inhibitor trametinib alone suggest single pathway suppression to be insufficient in reducing disease in MPNs. Compensatory mechanisms of reduced therapeutic efficacy likely include both adaptive upregulations of driver pathways such as increased levels of pSTAT1/3/5, pAKT, and pS6 effectors seen in HEL Fed-P cells as well as elevated intrinsic signaling driving disease transformation from chronic MPN phase to sAML. Recently, the merits of combinational therapy have been explored, with emphasis on concurrent inhibition of JAK/STAT and MAPK pathways. The addition of trametinib to ruxolitinib suppressed MEK/ERK feedback activation in Jak2 V617F and MPL W515L mice and reversed bone marrow fibrosis greater than in monotherapy(12). Similar therapeutic strategies also reduced disease penetrance and JAK2 V617F clonal burden in MPN xenograft models(11). Our findings with BCI indicate that DUSP6 targeting impacts MAPK in addition to multiple downstream signaling effectors. Continued investigation utilizing additional screening or synthetic lethal approaches may distinguish essential targets for rational therapeutic intervention.

Current understanding of disease transformation from chronic MPN to SAML is complicated by relationships between distinct cellular populations, diverse genomic alterations and temporal interactions. Our sequencing approaches led us to identify a handful of candidates, 
including DUSP6 which plays context-dependent oncogenic roles such as in glioblastoma, thyroid, and breast cancers, or tumor-suppressive roles in pancreatic, lung, and ovarian cancers(13-15). Our initial observation of aberrantly increased expression of DUSP6 specifically in SAML HSPCs suggested that DUSP6 may act as an oncogenic driver of leukemic progression. This was corroborated by functional studies including DUSP6 knockdown experiments which led to reduced viability of JAK2-mutant cells. The molecular mechanisms responsible for DUSP6 induction in the setting of MPN disease evolution are likely to be related to multiple factors, including underlying driver mutations and re-wiring of intracellular signaling pathways in the setting of JAK2 inhibition. Here, we provide proof-of-principle validation of regulatory transcriptional machinery co-mobilized with DUSP6 in KLF2 and KLF1. Further functional investigations of DUSP6 regulation may be warranted on other targets identified in this study such as EGR family proteins. Notably, we identified increased DUSP6 expression in de novo AML, suggesting that DUSP6 may be a converging downstream effector common to myeloid malignancies. Consistent with this notion, aberrant FLT3 expression was identified in our initial sAML transcriptome analysis, and FLT3-ITD (a common genetic alteration in de novo AML) has been shown to upregulate DUSP6 expression(61). These findings suggest that DUSP6 may represent a common, targetable dependency in AML.

$\mathrm{BCI}$ has been described to be an allosteric inhibitor of both DUSP1 and DUSP6(62) and by this canonical function, elevates ERK signaling. This was observed in vitro at low concentrations, but higher $\mathrm{BCl}$ doses abrogated $\mathrm{pERK}$, and was concomitant with increased apoptosis and cell cycle arrest. Further pSTAT and pS6 signaling inhibition upon BCI treatment suggest that dual inhibition of these effectors may contribute to the observed functional effects. Similar pathway suppression was also validated upon DUSP6 knockdown. These observations suggest that rather than off-target effects of $\mathrm{BCI}$, DUSP6 plays novel functions beyond direct regulation of ERK signaling which may be crucial to MPN/AML cell survival . BCl being able to suppress both DUSP1 and DUSP6 have also led to investigations of promiscuity. A recent study 
suggested a role for DUSP1 in protecting against DNA damage in PV progenitors via modulation of p38/JNK signaling(63). While we cannot exclude the possibility that our findings with $\mathrm{BCl}$ may be at least partially related to DUSP1 inhibition, our collective findings including bulk and single cell gene expression analyses, knockdown experiments, and mass cytometry profiling support a predominant role for DUSP6 regulation of MAPK/STAT/S6 phenotypes in leukemic transformation.

The efficacy of $\mathrm{BCl}$ has been briefly explored in other hematological malignancies. DUSP1/6 inhibition in combination with FOS inhibitor curcumin and imatinib suppressed tumor growth in BCR-ABL positive chronic myeloid leukemia $(C M L)(64)$. Notably in the context of CML, ectopic expression of DUSP1 but not DUSP6 conferred resistance to $\mathrm{BCl}$, and inhibitory proliferative effects were a consequence of DUSP1 inhibition, while DUSP6 inhibition favored CML cell survival upon tyrosine kinase inhibitor treatment. Dual pathway inhibition with $\mathrm{BCl}$ analog $\mathrm{BCl}-215$ in combination with ruxolitinib also demonstrated durable response in B-cell leukemia (B-ALL) models, where $\mathrm{BCl}-215$ substantially increased ERK phosphorylation, while combination treatment reduced pSTAT5 levels(65). Taken together with the findings from our study, these observations highlight the promising therapeutic potential of DUSP targeting, while reinforcing the notion that DUSP6 plays context-dependent roles even within specific hematologic malignancies. In these settings, the transduction interactome and precise signaling inhibition mechanisms of DUSP6 perturbation remain to be fully characterized.

We also identify RPS6KA1 as a clinically important marker in AML prognosis and key downstream target of DUSP6. We demonstrate that inhibition of RSK1 via BI-D1870 suppresses S6 signaling in vitro and ex vivo and exhibits combinatorial efficacy with BCI. BI-D1870 has recently been demonstrated to suppress AML proliferation in vitro(66), and a novel RSK inhibitor PMD-026 is currently being evaluated in a phase 1 clinical trial in breast cancer (NCT04115306) in addition to preclinical studies across other cancer types. Thus, our work provides a promising 
bioRxiv preprint doi: https://doi.org/10.1101/2021.06.27.449656; this version posted July 2, 2021. The copyright holder for this preprint (which was not certified by peer review) is the author/funder, who has granted bioRxiv a license to display the preprint in perpetuity. It is made available under aCC-BY-NC-ND 4.0 International license.

foundation for this novel therapeutic strategy targeting the DUSP6-RSK1-S6 axis in MPNs and AML. 


\section{Acknowledgements}

This work was supported by NIH grants R01HL134952 (S.T.O.), and T32HL007088 (J.S.F.). Technical support was provided by the Alvin J. Siteman Cancer Center Tissue Procurement Core Facility, Biostatistics Shared Resource, Flow Cytometry Core, and Immunomonitoring Laboratory, which are supported by NCl Cancer Center Support Grant P30CA91842. The Immunomonitoring Laboratory is also supported by the Andrew M and Jane M Bursky Center for Human Immunology and Immunotherapy Programs. We thank D. Bender, R. Lin, and K. Link for assistance with mass cytometry experiments. We are grateful to Ann Mullally for providing Jak2 V617F knock-in mice, and to Ross Levine for providing the MPL W515L retroviral construct. We thank Mary Fulbright for assistance with mouse colony management. We thank Tim Ley for sharing TCGA LAML data. We thank Feng Gao for assistance with biostatistical analysis. We thank Andreas Vogt for helpful discussions related to $\mathrm{BCl}$. We thank the Genetic Perturbation Service of Goodman Cancer Research Centre at McGill University for access to and preparation of functional genetic tools. G.A.C. was supported by NIH grant R01HL147978, the Edward P. Evans Foundation and the Gabrielle's Angel Foundation. G.A.C. is a scholar of the Leukemia and Lymphoma Society. This work was also supported by Canadian Institutes of Health Research (CIHR) grants PJT-156233 (S.H.) and PJT-438303 (S.H.). K.Y. is supported by a Canderel Rising Star Summer Studentship and S.H. is supported by a Canadian Research Chair in Functional Genomics.

\section{Author contributions}

T.K., A.B.B.L., K.Y., L.Y., D.A.F, and J.S.F. performed experiments. A.Z W., M.J.A., H.C., G.A.C., and S.T.O. provided technical and clinical support. M.R. evaluated histopathology. T.K., S.H., and S.T.O. designed and supervised the experiments. T.K. and S.T.O. wrote the manuscript. All authors read and approved of the manuscript. 


\section{Competing interests}

S.T.O. has served as a consultant for Kartos Therapeutics, CTI BioPharma, Celgene/Bristol Myers Squibb, Disc Medicine, Blueprint Medicines, PharmaEssentia, Constellation, Geron, Abbvie, Sierra Oncology, and Incyte. All other authors disclose no competing interests.

\section{Figure legends}

Figure 1. Elevated DUSP6 expression in patients with sAML compared to MPN.

A) Gene set enrichment analysis of top Hallmark pathways enriched and diminished in linage-negative CD34+ cells from sAML patient samples $(n=6)$ compared to MF $(n=14)$ from microarray analysis.

B) Volcano plot showing top elevated candidates in lineage-negative CD34+ cells from SAML patients compared to MF from microarray analysis. Dashed vertical lines denote boundaries of genes having at least 1 log2 fold change. Dashed horizontal line denotes p-value at $0.05(-\log 10(p)=1.3)$.

C) DUSP6 expression from CD34+ cells isolated from NBM $(n=5)$, MF $(n=14)$, and sAML $(n=6)$ patient samples. DUSP6 values represent RMA from microarray. Statistics were assessed by two-tailed Student's $t$ test.

D) DUSP6 expression from healthy, individual bone marrow mononuclear cells (NBM MNC, $n=19)$, prior-MPN sAML MNC ( $=24)$, prior-MDS sAML MNC $(n=45)$, and de novo AML MNC $(n=375)$ from the OHSU BeatAML cohort. DUSP6 values represent normalized RPKM. Statistics were assessed by two-tailed Student's $t$ test.

E) Immunofluorescence of bone marrow from MF, sAML, and healthy donors. White arrows denote DUSP6 positive cell staining. Scale bar: $50 \mu \mathrm{M}$. 
Figure 2. Serial patient CD34+ scRNA sequencing reveals elevated DUSP6 along MPN to sAML progression.

A) Schematic of scRNA-seq with Totalseq protein detection of 52,564 sorted CD34+ cells of serial samples from three MPN to SAML patients and two healthy bone marrow donors (N34, N39). Timeline shows disease stage and time (months) at which the sample was collected relative to early/first MPN stage.

B) UMAP clustering of CD34+ cells from chronic MPN and SAML patients, and healthy donors.

C) Venn diagram showing overlapping genes from the top 1,000 differentially expressed genes in serial CD34+ samples at the sAML stage compared to MPN stage of three patients (381812 MF sAML vs 38182 chronic MF, 145790 sAML VS 145790 early and late MF, 374024 sAML vs 374024 chronic PV).

D) Violin plot showing elevated DUSP6 expression at SAML stage compared to prior MPN stage. Bone marrow from two healthy donors (N34, N39) are included as references.

E) UMAP analysis of MF and SAML disease states (top) and identified cell populations (bottom) in patient 381812.

F) Violin plots of DUSP6, KLF1, and KLF2 across identified subpopulations in 381812 .

G) Trajectory analysis showing differentially expressed effectors DUSP6, KLF1, and $K L F 2$ at identified branch points in MF and SAML disease states (top) and identified cell populations (bottom) from patient 381812 .

H) Pseudotime analysis of dynamic DUSP6, KLF1, and KLF2 expression across disease progression from MF to sAML in patient 381812.

Figure 3. DUSP6 inhibition suppresses signaling from driver pathways and proliferation in MPN and AML. 
A) Immunoblot profiling of dominant signaling pathways in the JAK2 V617F mutant HEL cell line. HEL cells were treated with increasing doses of DUSP6 inhibitor $\mathrm{BCl}$ for 24 hours.

B) Cell cycle assay of $\mathrm{HEL}$ cells treated with $1 \mu \mathrm{M}$ of $\mathrm{BCl}$ or control for 12 hours. Cells were treated in triplicate $(n=3)$.

C) Annexin $\mathrm{V}$ apoptosis assay of $\mathrm{HEL}$ cells treated with $1 \mu \mathrm{M}$ of $\mathrm{BCl}$ or control for 48 and 72 hours. Cells were treated in triplicate $(n=3)$.

D) Hallmark gene set enrichment analysis of top altered pathways from RNA sequencing of HEL cells treated with $1 \mu \mathrm{M} \mathrm{BCl}$ or DMSO control for 4 and 24 hours.

E) Immunoblot of HEL cells upon knockdown of DUSP6 with two independent shRNAs or pLKO control vector.

F) Cell viability assay of HEL cells upon knockdown of DUSP6 with two independent shRNAs or pLKO control vector. Cells were plated at $n=6$ for each condition and grown for 96 hours with viability normalized to pLKO control vector. Mean and standard deviation presented. Statistics were assessed by two-tailed Student's t test.

G) Cell viability assay of normal donor bone marrow (NBM BMMC; $n=5$ ), and PBMCs from MPN $(n=8)$, and sAML $(n=11)$ patients. All primary cells were from unique patients and were treated for 72 hours with $150 \mathrm{nM} \mathrm{BCl}$. Statistics were assessed by two-tailed Student's t test.

H) Relative cleaved caspase 3 signal from CD34+ gated cells by mass cytometry. Unique sAML PBMCs $(n=7)$ and NBM BMMC $(n=3)$ were treated for 4 hours at $1 \mu \mathrm{M} \mathrm{BCl}$. Arcsinh ratios indicate relative change in the $\mathrm{BCl}$-treated condition compared to control. Statistics were assessed by two-tailed Mann-Whitney $U$ test.

I) Colony assay of sorted lin- CD34+ cells ( $n=3$ unique patients each for NBM, MF, and sAML). Sorted cells were grown in MethoCult H4034 Optimum for 12 days in $0.5 \mathrm{BCl} \mu \mathrm{M}$ or RPMI control. Samples were plated in duplicate. Statistics were assessed by Two-tailed Student's t test. 
J) Heatmap and dot plots of altered signaling pathways of lin- CD34+ cells from unique NBM BMMCs, and PBMCs from MF, and sAML patients by mass cytometry. Patient samples were treated with $1 \mu \mathrm{M} \mathrm{BCl}$ for 4 hours, $20 \mathrm{ng} / \mathrm{mL}$ TPO for 1 hour, or combination. Signals were normalized to the control treatment of each individual patient sample and reported as $90^{\text {th }}$ percentile Arcsinh ratio.

\section{Figure 4. DUSP6 regulates S6 signaling through indispensable RSK1.}

A) Relative RPS6KA1 expression across 804 pan-cancer cell lines from the Cancer Cell Line Encyclopedia.

B) Gene dependency score for RPS6KA1 CRISPR knock-out across 804 pan-cancer cell lines from the DepMap Portal.

C) Kaplan-Meier event free survival curve of patients stratified by RPS6KA1 expression from the TCGA LAML cohort $(n=173)$.

D) Kaplan-Meier overall survival curve of patients stratified by RPS6KA1 expression from the TCGA LAML cohort $(n=173)$

E) Cell viability curve of JAK2 V617F mutant cell lines HEL and UKE-1 treated with increasing concentrations of RSK1 inhibitor BI-D1870. Cells were treated for 72 hours and viability was normalized to the control treatment. $n=6$ replicates at each drug dose. Mean and standard deviation presented.

F) Immunoblot of HEL and UKE-1 cells treated with BI-D1870 or control for 24 hours.

G) Cell cycle assay of HEL cells treated with $5 \mu \mathrm{M}$ BI-D1870 or control for 12 and 24 hours. Cells were treated in triplicate $(n=3)$.

H) Annexin V apoptosis assay of HEL cells treated with $5 \mu \mathrm{M}$ of BI-D1870 or control for 48 and 72 hours. Cells were treated in triplicate $(n=3)$. 
I) Gene set enrichment analysis showing top altered pathways from the KEGG signaling and metabolism gene set from RNA sequencing of HEL cells treated with $10 \mu \mathrm{M}$ of BI-D1870 or control for 24 hours.

J) Top 50 differentially expressed genes from the KEGG PI3K-AKT signaling pathway of RNA-seq from HEL cells treated with $10 \mu \mathrm{M}$ of BI-D1870 for 24 hours vs control treatment.

K) Dot plots of altered signaling pathways of lin- CD34+ cells from unique normal bone marrow donors and peripheral blood of MF patients by mass cytometry. Patient samples were treated with $5 \mu \mathrm{M}$ BI-D1870 for 4 hours, 20ng/mL TPO for 1 hour, or combination. Signals were normalized to the control treatment of each individual patient sample and reported as $90^{\text {th }}$ percentile Arcsinh ratio.

L) Colony assays of sorted lin- CD34+ cells from unique normal bone marrow, MF and sAML patients. Sorted cells were grown in MethoCult H4034 Optimum for 12 days in $1.5 \mu \mathrm{M} \mathrm{BI-}$ D1870 or RPMI control. Samples were plated in duplicate.

M) Correlation of DUSP6 and RPS6KA1 from lin- CD34+ cells from MF $(n=14)$, and sAML $(n=6)$ patient samples. Expression values represent RMA from microarray.

N) Immunoblot of HEL and UKE-1 cells treated with $1 \mu \mathrm{M} \mathrm{BCl}$ or control for 24 hours.

O) Immunoblot of HEL cells after knockdown with two independent shRNAs targeting DUSP6 or non-targeting pLKO control

\section{Figure 5. DUSP6 inhibition overcomes persistence to JAK inhibitors.}

A) Cell viability assay of parental HEL, HEL ruxolitinib-persistent (Rux-P), and HEL fedratinibpersistent (Fed-P) cells treated at indicated doses of JAK2 inhibitors ruxolitinib and fedratinib. Cells were treated for 72 hours and viability was normalized to the control. $n=$ 6 replicates at each drug dose. Mean and standard deviation presented.

B) Fold change of DUSP6 mRNA expression in HEL persistent cells relative to HEL parental cells from RNA-seq analysis. 
C) Heatmap of a curated gene set of "DUSP6 target genes UP" from RNA-seq analysis of HEL parental, HEL Rux-P, and HEL Fed-P cells.

D) Cell viability assay of HEL parental cells treated with fedratinib and ruxolitinib after ectopic expression of DUSP6 or a control vector. Cells were treated with $0.625 \mu \mathrm{M}$ of JAK2 inhibitors for 96 hours and viability was normalized to the control treatment group from each population. $n=6$ replicates. Mean and standard deviation presented. Statistics were assessed by two-tailed Student's t test.

E) Immunoblot of HEL cells ectopically expressing DUSP6 or GFP control vector. Cells were treated with control, $1 \mu \mathrm{M} \mathrm{BCl}$, or $1 \mu \mathrm{M}$ fedratinib for 24 hours.

F) Cell viability assay of HEL parental cells treated with fedratinib after knockdown with two independent shRNAs targeting DUSP6 or non-targeting pLKO control. Cells were treated for 96 hours and viability was normalized to the control treatment group from each population. $n=5-6$ replicates at each drug dose. Mean and standard deviation presented. Statistics were assessed by two-way ANOVA with Dunnett's multiple comparisons test.

G) Cell viability assay of HEL parental cells treated with ruxolitinib after knockdown with two independent shRNAs targeting DUSP6 or non-targeting pLKO control. Cells were treated for 96 hours and viability was normalized to the control treatment group from each population. $n=6$ replicates at each drug dose. Mean and standard deviation presented. Statistics were assessed by two-way ANOVA with Dunnett's multiple comparisons test.

H) Expression profiling of key phospho-proteins in HEL parental and HEL Fed-P cells by mass cytometry. Expression denoted as median dual counts.

I) Mass cytometry heatmap of HEL Fed-P cells treated with $\mathrm{BCl}$, fedratinib, or combination. Cells were treated with $1 \mu \mathrm{M} \mathrm{BCl}$ and/or $1 \mu \mathrm{M}$ fedratinib for 1 hour and signals were normalized to the control treatment and reported as median Arcsinh ratio.

J) Schematic of disease progression and time course of clinical treatments of patient 973039 . 
K) Colony assays of sorted lin- CD34+ cells from 973039 at two timepoints, and healthy donor bone marrow (NBM 40). Sorted cells were grown in MethoCult H4034 Optimum for 2 weeks in the presence of inhibitors: Fedratinib panel: $1 \mu \mathrm{M}$ fedratinib; $\mathrm{BCl}$ panel: $0.5 \mu \mathrm{M}$ $\mathrm{BCl}$; 973039 T2 panel: $1 \mu \mathrm{M}$ fedratinib, $1 \mu \mathrm{M} \mathrm{BCl}$, and $1 \mu \mathrm{M}$ fedratinib $+1 \mu \mathrm{M} \mathrm{BCl}$. Samples were plated in triplicate. Statistics were assessed by two-tailed Student's t test.

L) Paired mass cytometry analysis of lin- CD34+ cells from 973039 T2 and NBM 40. Cells were treated with $1 \mu \mathrm{M} \mathrm{BCl}$ for 1 hour, $20 \mathrm{ng} / \mathrm{mL}$ TPO for 15 minutes, or combination.

\section{Figure 6. $\mathrm{BCl}$ is efficacious in MPN mouse models}

A) Schematic of the Jak2 mouse model. c-KIT+ cells from CD45.2 C57BL/6J mice were isolated and transplanted into CD45.1 C57BL/6J mice. Mice were treated with vehicle ( $\mathrm{n}$ = 9) or $25 \mathrm{mg} / \mathrm{kg} \mathrm{BCl}(\mathrm{n}=10)$ daily for 5 days followed by 2 days off treatment starting on day 17 .

B) Efficacy of $\mathrm{BCl}$ in the Jak2 mouse model. Plots show hematocrit and white blood cell (WBC) counts of transplanted mice treated with vehicle or $\mathrm{BCl}$ across multiple timepoints and and spleen and liver weights at endpoint. Hematocrit and WBC counts statistics were assessed by two-way ANOVA comparing vehicle and $\mathrm{BCl}$. Spleen and liver weights statistics were assessed by two-tailed Student's t test.

C) Schematic of the MPL mouse model. Retroviral MPL was transduced into c-KIT+ cells from CD45.2 C57BL/6J mice and transplanted into CD45.1 C57BL/6J mice. Mice were treated with vehicle $(n=8)$ or $25 \mathrm{mg} / \mathrm{kg} \mathrm{BCl}(\mathrm{n}=8)$ daily for 5 days followed by 2 days off treatment starting on day 17.

D) Efficacy of $\mathrm{BCl}$ in the MPL mouse model. Plots show hematocrit, WBC, and platelet counts of transplanted MPL mice treated with vehicle or $\mathrm{BCl}$ across multiple timepoints. WBC and platelet counts statistics were assessed by two-way ANOVA comparing vehicle and $\mathrm{BCl}$. 
E) Kaplan-Meier survival analysis of transplanted mice treated with vehicle or $\mathrm{BCl}$ were assessed by log-rank test.

F) Images show representative H\&E and reticulin staining of bone marrow of tibias from vehicle and $\mathrm{BCl}$ treated MPL mice at endpoint. Scale $=50 \mu \mathrm{M}$.

\section{Figure 7. $\mathrm{BCl}$ alleviates disease burden across sAML patient-derived xenograft models.}

A) Schematic of the CD34+ sAML patient-derived xenograft mouse models. CD34+ cells were isolated from patients SAML11 and SAML14 and transplanted into NSGS mice. For the sAML11 PDX, mice were treated with vehicle $(n=6)$ or $25 \mathrm{mg} / \mathrm{kg} \mathrm{BCl}(n=7)$ daily for 5 days followed by 2 days off treatment starting on day 24. For the sAML14 PDX, mice were treated with vehicle $(\mathrm{n}=7), 25 \mathrm{mg} / \mathrm{kg} \mathrm{BCl}(\mathrm{n}=8), 90 \mathrm{mg} / \mathrm{kg}$ ruxolitinib $(\mathrm{n}=7)$, or combination $(n=7)$ following weekly schedule of 5 days on, 2 days off treatment starting on day 31.

B) Efficacy of $\mathrm{BCl}$ in the sAML11 PDX model. Plots show percentage of human CD45 (hCD45) in peripheral blood and bone marrow of transplanted mice treated with vehicle or $\mathrm{BCl}$ across multiple timepoints and spleen weights of mice at endpoint normalized by mouse weight. \%hCD45 in PB statistics assessed by two-way ANOVA comparing vehicle to $\mathrm{BCl}$ \% \%CD45 in BM, and normalized spleen and liver weights statistics were assessed by two-tailed Student's t test.

C) Efficacy of treatment in the sAML14 PDX model. Plots show percentage of hCD45 cells in peripheral blood and bone marrow of transplanted mice treated with vehicle, $\mathrm{BCl}$, ruxolitinib, or combination across multiple timepoints. \%hCD45 in PB statistics were assessed by two-way ANOVA comparing vehicle vs each individual treatment group with Dunnett's multiple comparison test, and two-way ANOVA for ruxolitinib vs combination. \%hCD45 in BM statistics were assessed by one-way ANOVA with Dunnett's multiple comparison test. Rightmost panel: Mass cytometry of PBMCs isolated from the bone 
marrow of three sAML14 PDX mice from each treatment group at endpoint. Heatmap denotes row z-score calculated from mean signal intensity of each marker.

\section{Supplementary figure legends}

\section{Supplementary Figure 1. Elevated DUSP6 expression in patients with SAML compared to} MPN.

A) Heatmap of top differentially expressed genes from microarray analysis in lin- CD34+ cells from sAML patients $(n=14)$ compared to MF patients $(n=6)$, and reference expression in healthy donor bone marrow (NBM; $n=5)$.

B) DUSP6 expression from CD34+ cells from NBM $(n=31)$, AML bone marrow CD34+ subfraction $(n=46)$, and AML bone marrow CD34- subfraction $(n=44)$ from GSE30029. DUSP6 values represent quartile normalized, log-transformed values. Statistics were assessed by two-tailed Student's t test.

C) Immunofluorescence of bone marrow from additional MF and SAML patients, and healthy donors. White arrows denote DUSP6-positive cell staining. Scale bar: $50 \mu \mathrm{M}$.

D) Imaging mass cytometry analysis of PBMC cell pellets from normal donor peripheral blood (LRS2), MF (MF20), or sAML (sAML1) patients. Individual images show overlap of indicated channels as denoted. Scale bar $=16 \mu \mathrm{M}$.

\section{Supplementary Figure 2. Serial patient CD34+ scRNA sequencing shows elevated DUSP6 along MPN to sAML progression.}

A) Gene set enrichment analysis of top altered Hallmark pathways in serial CD34+ samples at the sAML stage compared to MPN stage of three patients.

B) Violin plots showing relative expression of top shared differentiate expressed genes in sAML vs MPN disease states from three patients, and healthy donors (N34, N39). 


\section{Supplementary Figure 3. Correlation between DUSP6 and top candidates identified along} disease progression.
A) Venn diagram showing shared candidates identified in the top 1000 differentially expressed genes from two sAML stages relative to their chronic MF stage(s).
B) Violin plots highlighting gene expression of shared transcription factors at SAML vs MF stage from from (a).
C) ChIP tracks of key transcription factors identified from scRNA-seq showing occupancy at the Mus musculus Dusp6 locus across various tissue samples.
D) Heatmap of pearson correlations between DUSP6 and top, shared candidates across five databases. ${ }^{*} P<0.05 ;{ }^{* *} P<0.01 ;{ }^{* * *} P<0.001 ;{ }^{* * *} P<0.0001$.
E) KLF2 identified as the top correlating gene, and KLF1 identified among the bottom 10 correlating genes, with DUSP6 in patient 381812 (MF and SAML) scRNA-seq.

\section{Supplementary Figure 4. Identification of distinct subpopulations from 381812 CD34+ scRNA-seq.}
A) TotalSeq surface protein detection and mRNA features to guide distinct subpopulation identification.
B) Schematic and relative quantification of distinct subpopulations identified from 381812 .

\section{Supplementary Figure 5. scRNA-seq subpopulation and trajectory analysis of additional} patients.

UMAP analysis and violin plot of DUSP6, KLF2, and $K L F 1$ of subpopulations from N34 (and N39) 374024 , and 145790 . Additional trajectory analysis of patients 374024 and 145790 along disease progression from MPN to sAML. 


\section{Supplementary Figure 6. Exploration of DUSP6 regulation by KLF1 and KLF2 across} additional models.

A) Schematic of a separate serial CD34+ scRNA-seq dataset of a primary MF patient at multiple timepoints along transformation to sAML. As per source publication by Parental et al., sample T1 (PMF) was collected at chronic MPN phase, after which the patient was treated with ruxolitinib for 8 months at which sample T2 (treatment PMF) was collected, and then after 11 months of ruxolitinib treatment at sAML diagnosis (T3; sAML).

B) Violin plots of DUSP6, KLF2, and KLF1 expression at different disease timepoints from Parenti et al. CD34+ scRNA-seq in (a).

C) Relative fold change of DUSP6, KLF2, and KLF1 expression at sAML timepoint (T3) compared to PMF (T1) in across identified cell populations from Parenti et al. CD34+ scRNA-seq in (a).

D) KLF2 (left) and KLF1 (right) expression from CD34+ cells from NBM $(n=5), M F(n=14)$, and sAML $(n=6)$ patient samples. DUSP6 values represent RMA from microarray. Statistics were assessed by two-tailed Student's t test.

E) qRT-PCR of DUSP6 after KLF2 knockdown in HEL cells. DUSP6 mRNA expression normalized to ACTB for each group and then normalized to pLKO vector control. $\mathrm{n}=3$ in each group.

F) Immunoblot of DUSP6 expression after KLF2 knockdown in HEL cells utilizing shRNA or pLKO control vector.

G) Cell viability assay of HEL cells after KLF2 knockdown relative to control vector. Cells were grown for 96 hours and viability was normalized to the pLKO control vector. $n=6$ replicates per construct. Mean and standard deviation presented. Statistics were assessed by twotailed Student's t test,

H) DUSP6 expression in KLF1 inducible iPSC-derived macrophages relative to control. Dataset investigated: GSE125150. 
I) KIf2 expression in myeloid-specific KIf2 knockout murine bone marrow derived macrophages. Dataset investigated: GSE149119.

J) Multi-Chip Significance score (S-score) of Dusp6 in KIf2 knockout murine embryonic yolk sac erythroid cells. Dataset investigated: GSE27602.

\section{Supplementary Figure 7. Functional characterization of DUSP6 in MPN/AML.}

A) Relative mRNA expression of DUSP family genes across 35 AML cell lines from the Cancer Cell Line Encyclopedia.

B) Relative mRNA expression of DUSP family genes in HEL cells from the Cancer Cell Line Encyclopedia.

C) Cell viability curves of $\mathrm{HEL}$ cells treated with $\mathrm{BCl}$ or trametinib across multiple drug doses. Cells were treated for 72 hours and viability was normalized to control. $n=6$ replicates at each drug dose. Mean and standard deviation presented.

D) Immunoblot of $\mathrm{HEL}$ cells treated with increasing doses of $\mathrm{BCl}$ or the $\mathrm{MEK}$ inhibitor trametinib. Cells were treated at their indicated drug dose for 24 hours.

E) Phospho-STAT3 and phospho-STAT5 flow cytometry of HEL cells treated with $1 \mu \mathrm{M}$ of $\mathrm{BCl}$ or control for 24 hours.

F) Immunoblot profiling of different signaling pathways altered by $\mathrm{BCl}$ and trametinib. $\mathrm{HEL}$ cells were treated with $1 \mu \mathrm{M}$ or $1 \mu \mathrm{M}$ trametinib for 24 hours.

G) Hallmark gene set enrichment analysis showing top altered pathways by normalized enrichment score (NES) and enrichment plots of E2F targets and G2M checkpoint from RNA-seq of HEL cells treated with $1 \mu \mathrm{M}$ of $\mathrm{BCl}$, or DMSO control for 24 hours.

H) Immunoblot of HEL cells ectopically expressing DUSP6 or GFP control vector.

I) Cell viability assay of HEL cells ectopically expressing DUSP6 or GFP control vector. Cells were plated at $n=6$ for each condition and grown for 96 hours with viability normalized to 
the control vector. Mean and standard deviation presented. Statistics were assessed by two-tailed Student's t test.

J) Cell viability assay of HEL cells ectopically expressing DUSP6 or GFP control vector treated with $300 \mathrm{nM} \mathrm{BCl}$. Cells were plated at $n=6$ for each condition and grown for 96 hours with viability from normalized to control treatment from each group. Mean and standard deviation presented. Statistics were assessed by two-tailed Student's t test,

K) Representative images of lin- CD34+ colonies grown in MethoCult H4034 Optimum for 12 days in $0.5 \mu \mathrm{M} \mathrm{BCl}$ or RPMI control. Samples plated in duplicate. Scale bar: $1000 \mu \mathrm{M}$.

\section{Supplementary Figure 8. Suppression of signaling and cytokine production in primary samples by $\mathrm{BCl}$ assessed by mass cytometry.}

A) tSNE dimensional reduction clustering of distinct subpopulations from sAML4 and altered signaling upon $\mathrm{BCl}$, TPO induction, or combination treatment post mass cytometry analysis. Samples were treated with $1 \mu \mathrm{M} \mathrm{BCl}$ for 4 hours, 20ng/mL TPO for 1 hour, or combination.

B) TPO-induced signaling across different subpopulations from sAML4. Patient samples were treated with $20 \mathrm{ng} / \mathrm{mL}$ TPO for 1 hour. Signals were normalized to the control treatment and reported as median Arcsinh ratio.

C) TPO-induced cytokine production across different subpopulations from SAML5. Patient samples were treated with $20 \mathrm{ng} / \mathrm{mL}$ TPO for 4 hour. Signals were normalized to the control treatment and reported as 90 percentile Arcsinh ratio.

D) Heatmap and dot plots of altered cytokine production of CD14+ monocytes from bone marrow (NBM40) and peripheral blood (NPB LRS2) of healthy donors, and PBMCs from MF and SAML patients by mass cytometry. Unique patient samples were treated with 1 $\mu \mathrm{M} \mathrm{BCl}$ for 4 hours, $20 \mathrm{ng} / \mathrm{mL}$ TPO for 4 hour, or combination. Signals were normalized to the control treatment of each individual patient sample and reported as $90^{\text {th }}$ percentile 
Arcsinh ratio. Basal cytokine expression in CD14+ cells from MF and sAML are also presented (left panel) and are normalized to the NBM/NPB within each individual CyTOF run to control for batch effect: run 1 - sAML4 and sAML6 normalized to NBM40; run 2 MF20 and MF102 normalized to NPB LRS2; run 3- MF40 and sAML5 normalized to NBM40.

E) Dot plot of MIP-1ß/CCL4 in CD123+ and CD16+ monocyte populations from sAML5. Samples were treated with $1 \mu \mathrm{M} \mathrm{BCl}$ for 4 hours, $20 \mathrm{ng} / \mathrm{mL}$ TPO for 4 hours, or combination. Signals were normalized to the control treatment and reported as 90 percentile Arcsinh ratio.

\section{Supplementary Figure 9. Functional characterization of RSK1 in MPN/AML.}

A) Heatmap of inhibitors of upstream regulators of S6 activity and the pearson correlation of their area under curve (AUC) with DUSP6 expression in AML cell lines.

B) CRISPR dropout screen showing RPS6KA1 as an essential gene in AML. Candidates were identified if meeting criteria of FDR $<10 \%$ and whose inhibition affected \# of $A M L$ lines but neither of non-AML lines. Data retrieved from Tzelepis et al.

C) RPS6KA1 expression across 10,071 patient samples representing 31 distinct cancer subtypes from the TCGA Pan-Cancer cohort. Expression values provided as log2 (value +1). See additional information in methods.

D) Western blot analysis of RPS6KA1 knockdown by shRNA or control vector in HEL cells.

E) Cell viability assay of HEL cells after RPS6KA1 knockdown relative to control vector. Cells were grown for 96 hours and viability was normalized to the pLKO control vector. $n=6$ replicates per construct. Mean and standard deviation presented. Statistics were assessed by two-tailed Student's t test,

F) Heatmap of altered signaling pathways of lin- CD34+ cells from unique normal bone marrow donors and peripheral blood of MF patients by mass cytometry. Patient samples 
were treated with $5 \mu \mathrm{M} \mathrm{BI-D1870}$ for 4 hours, 20ng/mL TPO for 1 hour, or combination. Signals were normalized to the control treatment of each individual patient sample and reported as $90^{\text {th }}$ percentile Arcsinh ratio.

G) Ridge plot of RPS6KA1 expression from CD34+ scRNA-seq of N34, N39, and 381812 at MF and sAML stages.

H) RPS6KA1 expression from CD34+ cells from NBM $(n=5), M F(n=14)$, and sAML $(n=6)$ patient samples. RPS6KA1 values represent RMA from microarray. Statistics were assessed by two-tailed Student's t test.

I) Cell viability curve of HEL cells after ectopic expression of RPS6KA1 or GFP control treated with increasing concentrations of BI-D1870. Cells were treated for 96 hours and viability was normalized to the control treatment from each group. $n=6$ replicates per construct. Mean and standard deviation presented.

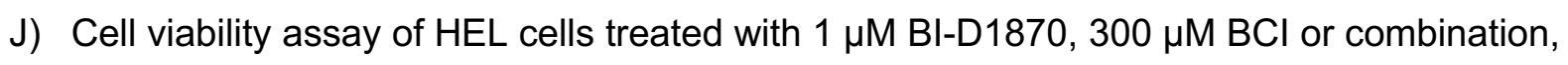
and UKE-1 cells treated with $2 \mu \mathrm{M}$ BI-D1870, $200 \mu \mathrm{M} \mathrm{BCl}$, or combination. Cells were treated for 72 hours and viability was normalized to the control treatment. $n=6$ replicates at each drug dose. Mean and standard deviation presented.

K) Hallmark gene set enrichment analysis showing top altered pathways from RNA-seq of HEL cells treated for 24 hours with $10 \mu \mathrm{M}$ BI-D1870 + $1 \mu \mathrm{M} \mathrm{BCl}$ compared to DMSO control (left) and $10 \mu \mathrm{M}$ BI-D1870 + $1 \mu \mathrm{M} \mathrm{BCl}$ compared to $10 \mu \mathrm{M}$ BI-D1870 alone (right).

L) Dot plot of mass cytometry analysis of lin- CD34+ cells from MF103 treated with $1 \mu \mathrm{M} \mathrm{BCl}$ for 4 hours, $5 \mu \mathrm{M} \mathrm{BI-D1870} \mathrm{for} 4$ hours, 20ng/mL TPO for 1 hour, or combination. Signals of key phosphorylated proteins were normalized to the control treatment and reported as $90^{\text {th }}$ percentile Arcsinh ratio.

\section{Supplementary Figure 10. DUSP6 mediates response to JAK2 inhibitors.}


A) Correlation of DUSP6 expression and fedratinib $I_{50}$ in $18 \mathrm{AML}$ cell lines. DUSP6 expression obtained from the CCLE database and fedratinib $\mathrm{IC}_{50}$ obtained from the GDSC1 collection.

B) Cell viability assay of HEL parental or HEL Fed-P cells treated with $\mathrm{BCl}$ at the indicated doses. Cells were treated for 72 hours and viability was normalized to the control treatment from each group. $n=6$ replicates at each drug dose. Mean and standard deviation presented.

C) Cell viability assay of $\mathrm{HEL}$ cells treated with $\mathrm{BCl}$, fedratinib, or combination. Cells were treated for 72 hours at the indicated drug doses and viability was normalized to the control treatment. $\mathrm{n}=6$ replicates at each drug dose. Mean and standard deviation presented.

\section{Supplementary Figure 11. BCl alleviates disease burden across MPN and SAML mouse models.}

A) WBC subpopulation counts, platelet counts, and body weight from Jak2 transplanted mice treated with vehicle or $\mathrm{BCl}$ across multiple timepoints. Statistics were assessed by twoway ANOVA comparing vehicle to $\mathrm{BCl}$.

B) Representative gross spleen of Jak2 mice treated with vehicle or $\mathrm{BCl}$ at endpoint.

C) Hematocrit, white blood cell (WBC) counts and differentials, and platelets counts of wildtype primary mice treated with vehicle $(n=4)$ or $25 \mathrm{mg} / \mathrm{kg} \mathrm{BCl}(n=5)$ following weekly schedule of 5 days on, 2 days off treatment across multiple timepoints. Liver, spleen, and body weights collected at endpoint. Two-way ANOVA and two-tailed Student's $t$ test statistical analysis resulted in non-significant values across all comparisons between vehicle and $\mathrm{BCl}$ treatment.

D) Representative flow cytometry analysis of peripheral blood from CD45.1 mice showing engraftment of MPL W515 GFP+ CD45.2 cells. 
E) WBC subpopulation counts, platelet counts, and normalized spleen weight from MPL W515 MF model of transplanted mice treated with vehicle or $\mathrm{BCl}$ across multiple timepoints. Statistics were assessed by two-way ANOVA for white count differential comparisons between vehicle and $\mathrm{BCl}$ and two-tailed Student's $\mathrm{t}$ test for normalized spleen weight.

F) Normalized spleen and liver weights from mice at end-point from sAML14 CD34+ PDX.

G) tSNE dimensional reduction clustering of mouse and human CD45+ cells from bone marrow of sAML14 PDX mice. 


\section{$\underline{\text { References }}$}

1. Levine RL, Gilliland DG. Myeloproliferative disorders. Blood 2008;112(6):2190-8 doi 10.1182/blood-2008-03-077966.

2. Oh ST, Gotlib J. JAK2 V617F and beyond: role of genetics and aberrant signaling in the pathogenesis of myeloproliferative neoplasms. Expert Rev Hematol 2010;3(3):323-37 doi 10.1586/ehm.10.28.

3. Zoi K, Cross NC. Genomics of Myeloproliferative Neoplasms. J Clin Oncol 2017;35(9):947-54 doi 10.1200/JCO.2016.70.7968.

4. Tefferi A. Myelofibrosis with myeloid metaplasia. N Engl J Med 2000;342(17):1255-65 doi 10.1056/NEJM200004273421706.

5. Harrison C, Kiladjian JJ, Al-Ali HK, Gisslinger H, Waltzman R, Stalbovskaya V, et al. JAK inhibition with ruxolitinib versus best available therapy for myelofibrosis. $\mathrm{N}$ Engl J Med 2012;366(9):787-98 doi 10.1056/NEJMoa1110556.

6. Verstovsek S, Mesa RA, Gotlib J, Levy RS, Gupta V, DiPersio JF, et al. A double-blind, placebo-controlled trial of ruxolitinib for myelofibrosis. N Engl J Med 2012;366(9):799807 doi 10.1056/NEJMoa1110557.

7. Vannucchi AM, Lasho TL, Guglielmelli P, Biamonte F, Pardanani A, Pereira A, et al. Mutations and prognosis in primary myelofibrosis. Leukemia 2013;27(9):1861-9 doi 10.1038/leu.2013.119.

8. Dunbar AJ, Rampal RK, Levine R. Leukemia secondary to myeloproliferative neoplasms. Blood 2020;136(1):61-70 doi 10.1182/blood.2019000943.

9. Patel AA, Odenike O. Genomics of MPN progression. Hematology Am Soc Hematol Educ Program 2020;2020(1):440-9 doi 10.1182/hematology.2020000129.

10. Rampal R, Ahn J, Abdel-Wahab O, Nahas M, Wang K, Lipson D, et al. Genomic and functional analysis of leukemic transformation of myeloproliferative neoplasms. Proc Natl Acad Sci U S A 2014;111(50):E5401-10 doi 10.1073/pnas.1407792111.

11. Jayavelu AK, Schnoder TM, Perner F, Herzog C, Meiler A, Krishnamoorthy G, et al. Splicing factor YBX1 mediates persistence of JAK2-mutated neoplasms. Nature 2020;588(7836):157-63 doi 10.1038/s41586-020-2968-3.

12. Stivala S, Codilupi T, Brkic S, Baerenwaldt A, Ghosh N, Hao-Shen H, et al. Targeting compensatory MEK/ERK activation increases JAK inhibitor efficacy in myeloproliferative neoplasms. J Clin Invest 2019;129(4):1596-611 doi 10.1172/JCI98785.

13. Keyse SM. Dual-specificity MAP kinase phosphatases (MKPs) and cancer. Cancer Metastasis Rev 2008;27(2):253-61 doi 10.1007/s10555-008-9123-1.

14. Bermudez O, Pages G, Gimond C. The dual-specificity MAP kinase phosphatases: critical roles in development and cancer. Am J Physiol Cell Physiol 2010;299(2):C189202 doi 10.1152/ajpcell.00347.2009.

15. Ahmad MK, Abdollah NA, Shafie NH, Yusof NM, Razak SRA. Dual-specificity phosphatase 6 (DUSP6): a review of its molecular characteristics and clinical relevance in cancer. Cancer Biol Med 2018;15(1):14-28 doi 10.20892/j.issn.2095-3941.2017.0107.

16. Ritchie ME, Phipson B, Wu D, Hu Y, Law CW, Shi W, et al. limma powers differential expression analyses for RNA-sequencing and microarray studies. Nucleic Acids Res 2015;43(7):e47 doi 10.1093/nar/gkv007.

17. Dobin A, Davis CA, Schlesinger F, Drenkow J, Zaleski C, Jha S, et al. STAR: ultrafast universal RNA-seq aligner. Bioinformatics 2013;29(1):15-21 doi 10.1093/bioinformatics/bts635.

18. Robinson MD, McCarthy DJ, Smyth GK. edgeR: a Bioconductor package for differential expression analysis of digital gene expression data. Bioinformatics 2010;26(1):139-40 doi 10.1093/bioinformatics/btp616. 
19. Luo W, Friedman MS, Shedden K, Hankenson KD, Woolf PJ. GAGE: generally applicable gene set enrichment for pathway analysis. BMC Bioinformatics 2009;10:161 doi 10.1186/1471-2105-10-161.

20. Subramanian A, Tamayo P, Mootha VK, Mukherjee S, Ebert BL, Gillette MA, et al. Gene set enrichment analysis: a knowledge-based approach for interpreting genome-wide expression profiles. Proc Natl Acad Sci U S A 2005;102(43):15545-50 doi 10.1073/pnas.0506580102.

21. Satija R, Farrell JA, Gennert D, Schier AF, Regev A. Spatial reconstruction of single-cell gene expression data. Nat Biotechnol 2015;33(5):495-502 doi 10.1038/nbt.3192.

22. Tirosh I, Izar B, Prakadan SM, Wadsworth MH, 2nd, Treacy D, Trombetta JJ, et al. Dissecting the multicellular ecosystem of metastatic melanoma by single-cell RNA-seq. Science 2016;352(6282):189-96 doi 10.1126/science.aad0501.

23. Korotkevich G, Sukhov V, Budin N, Shpak B, Artyomov MN, Sergushichev A. Fast gene set enrichment analysis. bioRxiv 2021:060012 doi 10.1101/060012.

24. Qiu X, Mao Q, Tang Y, Wang L, Chawla R, Pliner HA, et al. Reversed graph embedding resolves complex single-cell trajectories. Nat Methods 2017;14(10):979-82 doi 10.1038/nmeth.4402.

25. Fisher DAC, Miner CA, Engle EK, Hu H, Collins TB, Zhou A, et al. Cytokine production in myelofibrosis exhibits differential responsiveness to JAK-STAT, MAP kinase, and NFkappaB signaling. Leukemia 2019;33(8):1978-95 doi 10.1038/s41375-019-0379-y.

26. Bendall SC, Simonds EF, Qiu P, Amir el AD, Krutzik PO, Finck R, et al. Single-cell mass cytometry of differential immune and drug responses across a human hematopoietic continuum. Science 2011;332(6030):687-96 doi 10.1126/science.1198704.

27. Fisher DAC, Malkova O, Engle EK, Miner CA, Fulbright MC, Behbehani GK, et al. Mass cytometry analysis reveals hyperactive NF Kappa B signaling in myelofibrosis and secondary acute myeloid leukemia. Leukemia 2017;31(9):1962-74 doi 10.1038/leu.2016.377.

28. Bandyopadhyay S, Fowles JS, Yu L, Fisher DAC, Oh ST. Identification of functionally primitive and immunophenotypically distinct subpopulations in secondary acute myeloid leukemia by mass cytometry. Cytometry B Clin Cytom 2019;96(1):46-56 doi 10.1002/cyto.b.21743.

29. Bandyopadhyay S, Fisher DAC, Malkova O, Oh ST. Analysis of Signaling Networks at the Single-Cell Level Using Mass Cytometry. Methods Mol Biol 2017;1636:371-92 doi 10.1007/978-1-4939-7154-1_24.

30. Catena R, Montuenga LM, Bodenmiller B. Ruthenium counterstaining for imaging mass cytometry. J Pathol 2018;244(4):479-84 doi 10.1002/path.5049.

31. Kelly LM, Liu Q, Kutok JL, Williams IR, Boulton CL, Gilliland DG. FLT3 internal tandem duplication mutations associated with human acute myeloid leukemias induce myeloproliferative disease in a murine bone marrow transplant model. Blood 2002;99(1):310-8 doi 10.1182/blood.v99.1.310.

32. Celik H, Koh WK, Kramer AC, Ostrander EL, Mallaney C, Fisher DAC, et al. JARID2 Functions as a Tumor Suppressor in Myeloid Neoplasms by Repressing Self-Renewal in Hematopoietic Progenitor Cells. Cancer Cell 2018;34(5):741-56 e8 doi 10.1016/j.ccell.2018.10.008.

33. Celik H, Krug E, Zhang CR, Han W, Issa N, Koh WK, et al. A Humanized Animal Model Predicts Clonal Evolution and Therapeutic Vulnerabilities in Myeloproliferative Neoplasms. bioRxiv 2020:2020.11.12.378810 doi 10.1101/2020.11.12.378810.

34. Tyner JW, Tognon CE, Bottomly D, Wilmot B, Kurtz SE, Savage SL, et al. Functional genomic landscape of acute myeloid leukaemia. Nature 2018;562(7728):526-31 doi 10.1038/s41586-018-0623-z. 
35. de Jonge HJ, Woolthuis CM, Vos AZ, Mulder A, van den Berg E, Kluin PM, et al. Gene expression profiling in the leukemic stem cell-enriched CD34+ fraction identifies target genes that predict prognosis in normal karyotype AML. Leukemia 2011;25(12):1825-33 doi 10.1038/leu.2011.172.

36. Norfo R, Zini R, Pennucci V, Bianchi E, Salati S, Guglielmelli P, et al. miRNA-mRNA integrative analysis in primary myelofibrosis CD34+ cells: role of miR-155/JARID2 axis in abnormal megakaryopoiesis. Blood 2014;124(13):e21-32 doi 10.1182/blood-2013-12544197.

37. Cancer Genome Atlas Research N, Ley TJ, Miller C, Ding L, Raphael BJ, Mungall AJ, et al. Genomic and epigenomic landscapes of adult de novo acute myeloid leukemia. N Engl J Med 2013;368(22):2059-74 doi 10.1056/NEJMoa1301689.

38. Cerami E, Gao J, Dogrusoz U, Gross BE, Sumer SO, Aksoy BA, et al. The cBio cancer genomics portal: an open platform for exploring multidimensional cancer genomics data. Cancer Discov 2012;2(5):401-4 doi 10.1158/2159-8290.CD-12-0095.

39. Tzelepis K, Koike-Yusa H, De Braekeleer E, Li Y, Metzakopian E, Dovey OM, et al. A CRISPR Dropout Screen Identifies Genetic Vulnerabilities and Therapeutic Targets in Acute Myeloid Leukemia. Cell Rep 2016;17(4):1193-205 doi 10.1016/j.celrep.2016.09.079.

40. Meyers RM, Bryan JG, McFarland JM, Weir BA, Sizemore AE, Xu H, et al. Computational correction of copy number effect improves specificity of CRISPR-Cas 9 essentiality screens in cancer cells. Nat Genet 2017;49(12):1779-84 doi 10.1038/ng.3984.

41. Barretina J, Caponigro G, Stransky N, Venkatesan K, Margolin AA, Kim S, et al. The Cancer Cell Line Encyclopedia enables predictive modelling of anticancer drug sensitivity. Nature 2012;483(7391):603-7 doi 10.1038/nature11003.

42. Yang W, Soares J, Greninger P, Edelman EJ, Lightfoot H, Forbes S, et al. Genomics of Drug Sensitivity in Cancer (GDSC): a resource for therapeutic biomarker discovery in cancer cells. Nucleic Acids Res 2013;41(Database issue):D955-61 doi 10.1093/nar/gks1111.

43. Parenti S, Rontauroli S, Carretta C, Mallia S, Genovese E, Chiereghin C, et al. Mutated clones driving leukemic transformation are already detectable at the single-cell level in CD34-positive cells in the chronic phase of primary myelofibrosis. NPJ Precis Oncol 2021;5(1):4 doi 10.1038/s41698-021-00144-9.

44. Lopez-Yrigoyen M, Yang CT, Fidanza A, Cassetta L, Taylor AH, McCahill A, et al. Genetic programming of macrophages generates an in vitro model for the human erythroid island niche. Nat Commun 2019;10(1):881 doi 10.1038/s41467-019-08705-0.

45. Sweet DR, Vasudevan NT, Fan L, Booth CE, Keerthy KS, Liao X, et al. Myeloid Kruppellike factor 2 is a critical regulator of metabolic inflammation. Nat Commun 2020;11(1):5872 doi 10.1038/s41467-020-19760-3.

46. Redmond LC, Dumur Cl, Archer KJ, Grayson DR, Haar JL, Lloyd JA. Kruppel-like factor 2 regulated gene expression in mouse embryonic yolk sac erythroid cells. Blood Cells Mol Dis 2011;47(1):1-11 doi 10.1016/j.bcmd.2011.03.002.

47. Fang B, Everett LJ, Jager J, Briggs E, Armour SM, Feng D, et al. Circadian enhancers coordinate multiple phases of rhythmic gene transcription in vivo. Cell 2014;159(5):114052 doi 10.1016/j.cell.2014.10.022.

48. Tong AJ, Liu X, Thomas BJ, Lissner MM, Baker MR, Senagolage MD, et al. A Stringent Systems Approach Uncovers Gene-Specific Mechanisms Regulating Inflammation. Cell 2016;165(1):165-79 doi 10.1016/j.cell.2016.01.020.

49. Eichenfield DZ, Troutman TD, Link VM, Lam MT, Cho H, Gosselin D, et al. Tissue damage drives co-localization of NF-kappaB, Smad3, and Nrf2 to direct Rev-erb sensitive wound repair in mouse macrophages. Elife 2016;5 doi 10.7554/eLife.13024. 
50. Ciofani M, Madar A, Galan C, Sellars M, Mace K, Pauli F, et al. A validated regulatory network for Th17 cell specification. Cell 2012;151(2):289-303 doi 10.1016/j.cell.2012.09.016.

51. Link VM, Duttke SH, Chun HB, Holtman IR, Westin E, Hoeksema MA, et al. Analysis of Genetically Diverse Macrophages Reveals Local and Domain-wide Mechanisms that Control Transcription Factor Binding and Function. Cell 2018;173(7):1796-809 e17 doi 10.1016/j.cell.2018.04.018.

52. Shen Y, Yue F, McCleary DF, Ye Z, Edsall L, Kuan S, et al. A map of the cis-regulatory sequences in the mouse genome. Nature 2012;488(7409):116-20 doi 10.1038/nature11243.

53. Daver N, Schlenk RF, Russell NH, Levis MJ. Targeting FLT3 mutations in AML: review of current knowledge and evidence. Leukemia 2019;33(2):299-312 doi 10.1038/s41375018-0357-9.

54. Edwards DKt, Watanabe-Smith K, Rofelty A, Damnernsawad A, Laderas T, Lamble A, et al. CSF1R inhibitors exhibit antitumor activity in acute myeloid leukemia by blocking paracrine signals from support cells. Blood 2019;133(6):588-99 doi 10.1182/blood-201803-838946.

55. Testa U, Riccioni R, Militi S, Coccia E, Stellacci E, Samoggia P, et al. Elevated expression of IL-3Ralpha in acute myelogenous leukemia is associated with enhanced blast proliferation, increased cellularity, and poor prognosis. Blood 2002;100(8):2980-8 doi 10.1182/blood-2002-03-0852.

56. Bialkowska AB, Yang VW, Mallipattu SK. Kruppel-like factors in mammalian stem cells and development. Development 2017;144(5):737-54 doi 10.1242/dev.145441.

57. Ke J, Gururajan M, Kumar A, Simmons A, Turcios L, Chelvarajan RL, et al. The role of MAPKs in B cell receptor-induced down-regulation of Egr-1 in immature B lymphoma cells. J Biol Chem 2006;281(52):39806-18 doi 10.1074/jbc.M604671200.

58. Furukawa T, Kanai N, Shiwaku HO, Soga N, Uehara A, Horii A. AURKA is one of the downstream targets of MAPK1/ERK2 in pancreatic cancer. Oncogene 2006;25(35):4831-9 doi 10.1038/sj.onc.1209494.

59. Mullally A, Lane SW, Ball B, Megerdichian C, Okabe R, Al-Shahrour F, et al. Physiological Jak2V617F expression causes a lethal myeloproliferative neoplasm with differential effects on hematopoietic stem and progenitor cells. Cancer Cell 2010;17(6):584-96 doi 10.1016/j.ccr.2010.05.015.

60. Pikman Y, Lee BH, Mercher T, McDowell E, Ebert BL, Gozo M, et al. MPLW515L is a novel somatic activating mutation in myelofibrosis with myeloid metaplasia. PLoS Med 2006;3(7):e270 doi 10.1371/journal.pmed.0030270.

61. Arora D, Kothe S, van den Eijnden M, Hooft van Huijsduijnen R, Heidel F, Fischer T, et al. Expression of protein-tyrosine phosphatases in Acute Myeloid Leukemia cells: FLT3 ITD sustains high levels of DUSP6 expression. Cell Commun Signal 2012;10(1):19 doi 10.1186/1478-811X-10-19.

62. Molina G, Vogt A, Bakan A, Dai W, Queiroz de Oliveira P, Znosko W, et al. Zebrafish chemical screening reveals an inhibitor of Dusp6 that expands cardiac cell lineages. Nat Chem Biol 2009;5(9):680-7 doi 10.1038/nchembio.190.

63. Stetka J, Vyhlidalova P, Lanikova L, Koralkova P, Gursky J, Hlusi A, et al. Addiction to DUSP1 protects JAK2V617F-driven polycythemia vera progenitors against inflammatory stress and DNA damage, allowing chronic proliferation. Oncogene 2019;38(28):5627-42 doi 10.1038/s41388-019-0813-7.

64. Kesarwani M, Kincaid Z, Gomaa A, Huber E, Rohrabaugh S, Siddiqui Z, et al. Targeting c-FOS and DUSP1 abrogates intrinsic resistance to tyrosine-kinase inhibitor therapy in BCR-ABL-induced leukemia. Nat Med 2017;23(4):472-82 doi 10.1038/nm.4310. 
bioRxiv preprint doi: https://doi.org/10.1101/2021.06.27.449656; this version posted July 2, 2021. The copyright holder for this preprint (which

was not certified by peer review) is the author/funder, who has granted bioRxiv a license to display the preprint in perpetuity. It is made available under aCC-BY-NC-ND 4.0 International license.

65. Chan LN, Murakami MA, Robinson ME, Caeser R, Sadras T, Lee J, et al. Signalling input from divergent pathways subverts $B$ cell transformation. Nature 2020;583(7818):845-51 doi 10.1038/s41586-020-2513-4.

66. Chae HD, Dutta R, Tiu B, Hoff FW, Accordi B, Serafin V, et al. RSK inhibitor BI-D1870 inhibits acute myeloid leukemia cell proliferation by targeting mitotic exit. Oncotarget 2020;11(25):2387-403 doi 10.18632/oncotarget.27630. 

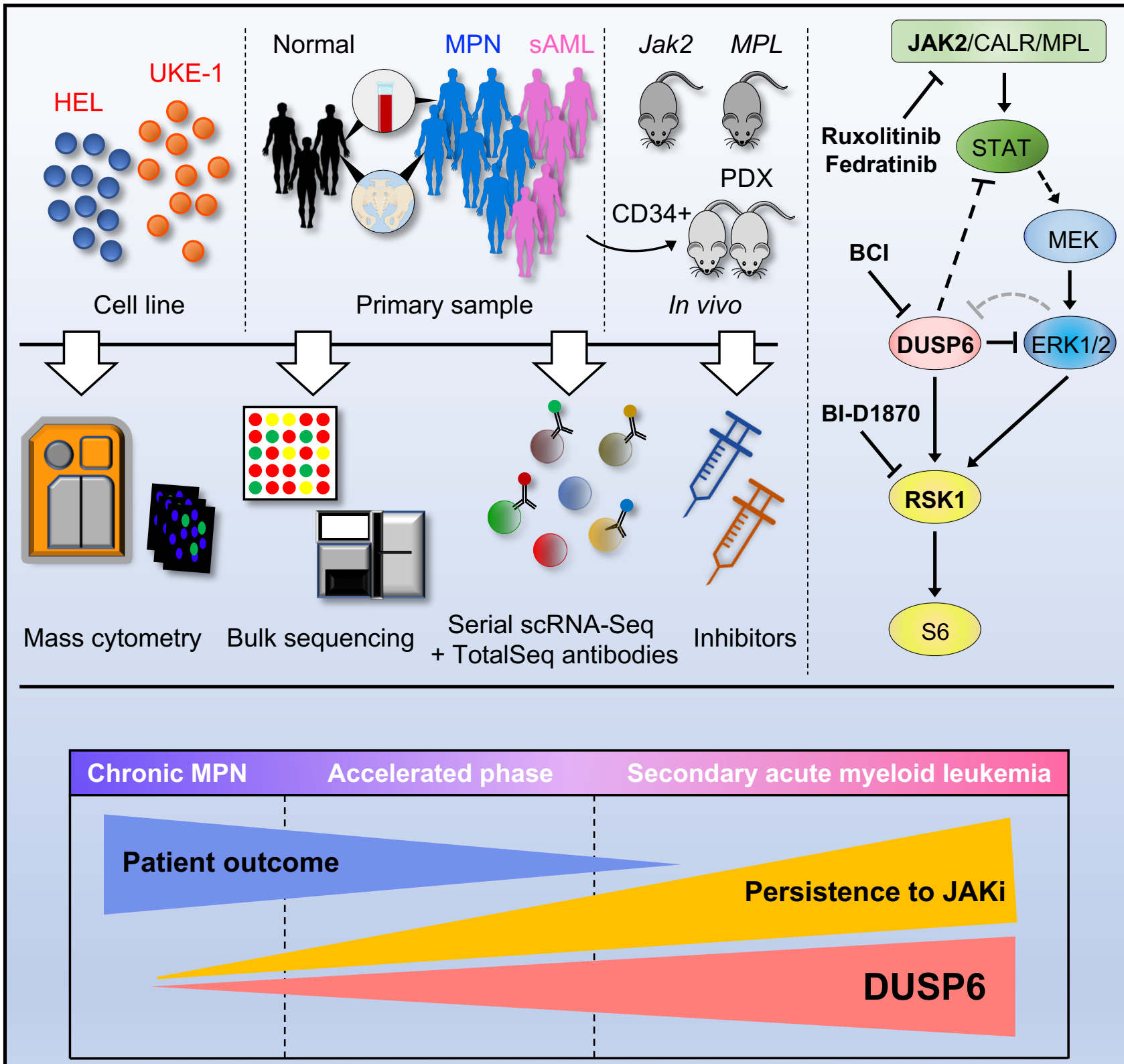

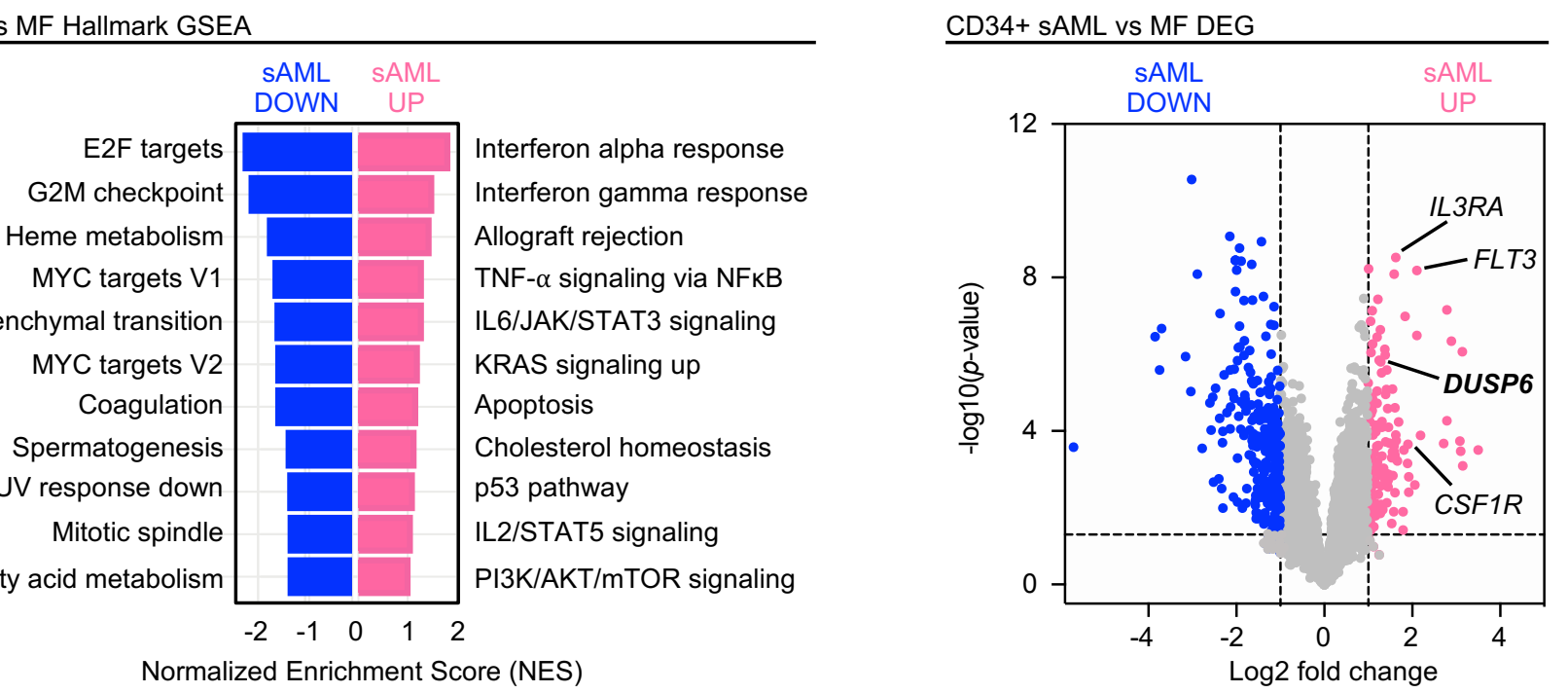

C

\section{E}
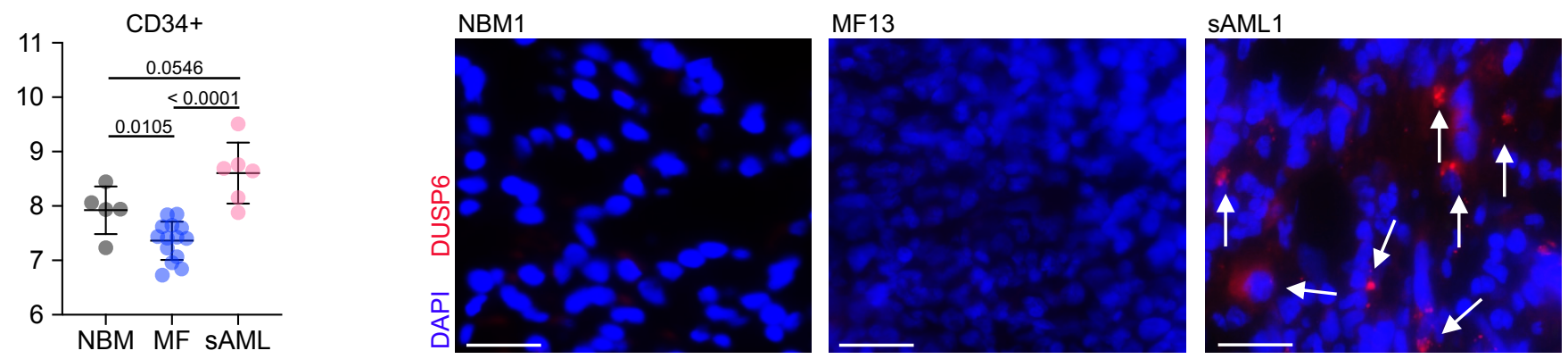

D

OHSU BeatAML

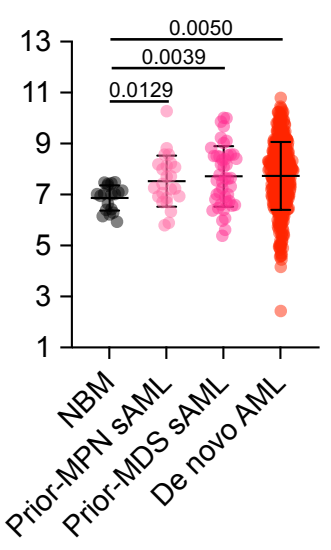

NBM5

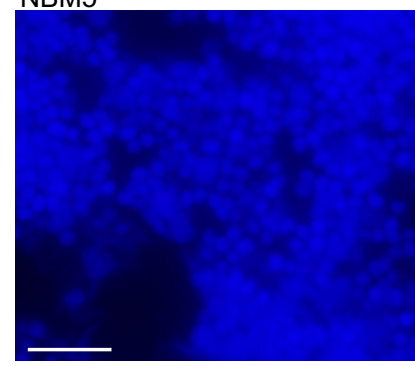

MF 381812 Day 0

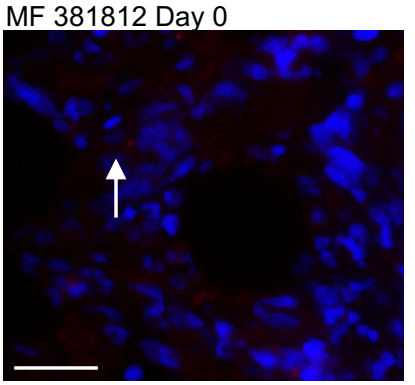

MF 381812 Day 245

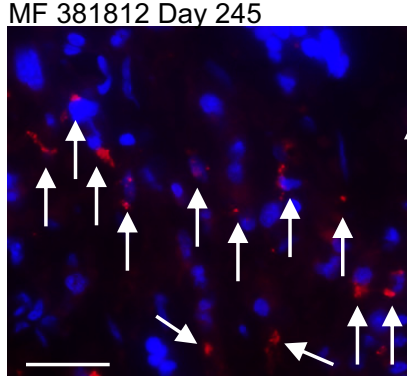


A

RPS6KA1 expression

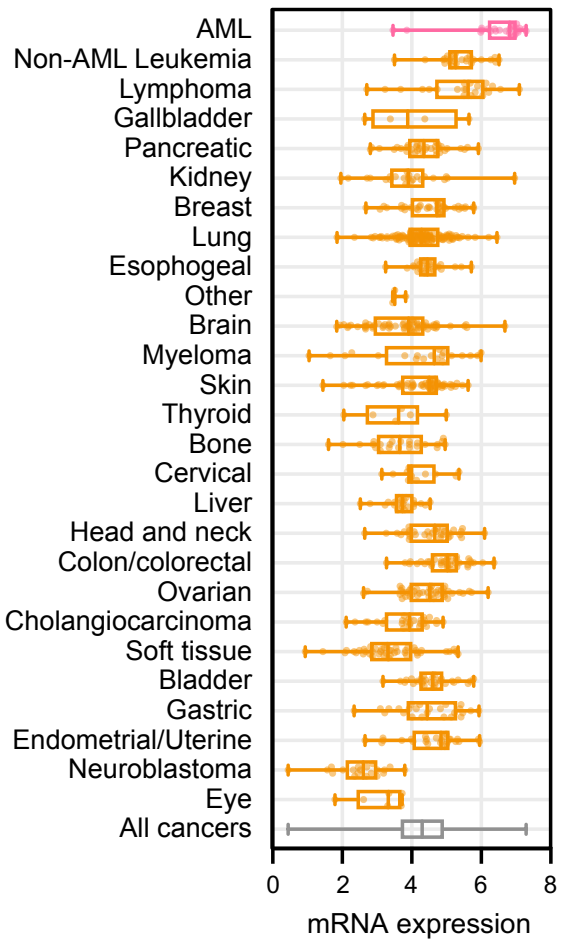

C

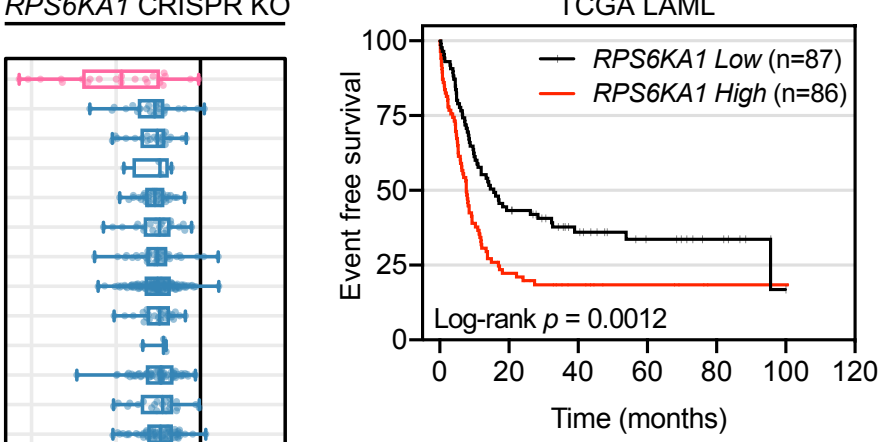

Figure 4
RPS6KA1 CRISPR KO

B

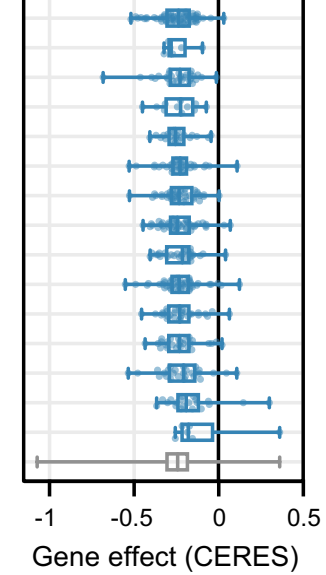

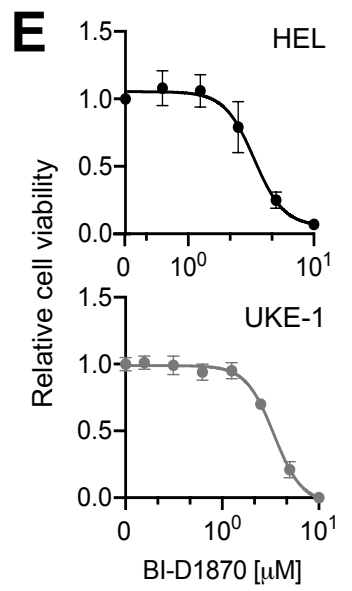

K

Lin- CD34+

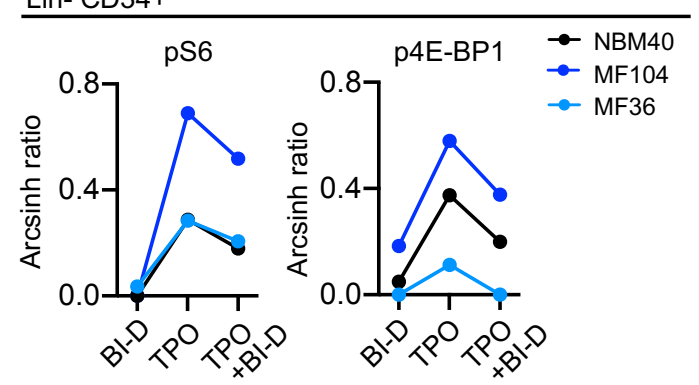

M

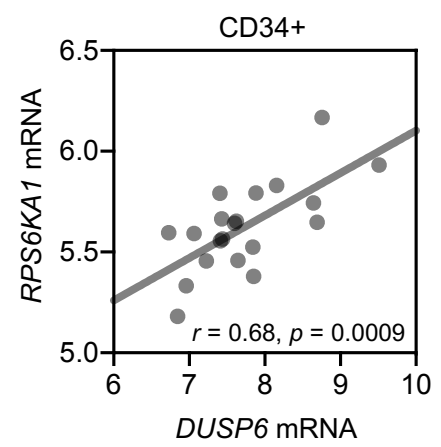

N
D
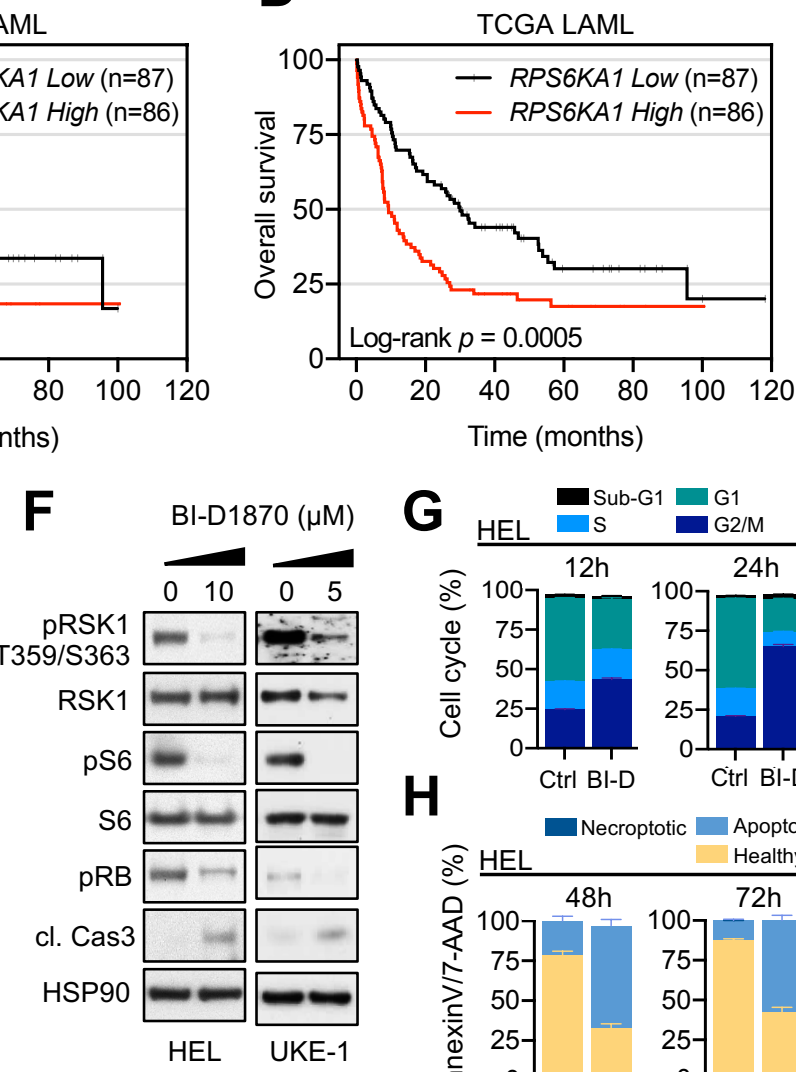

L
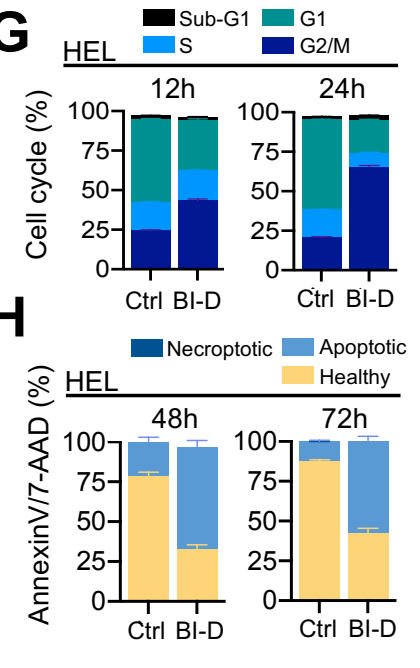

CD34+ BI-D1870 $\square$ Erythroid $\square$ Myeloid

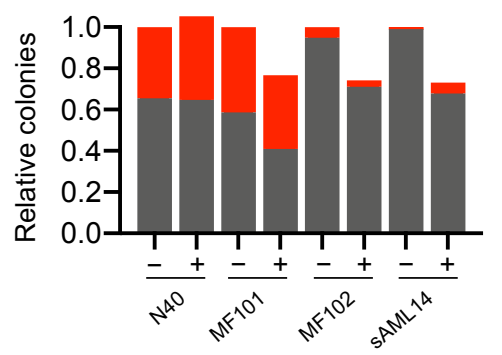

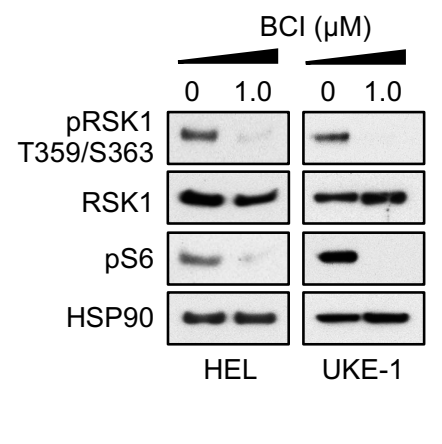

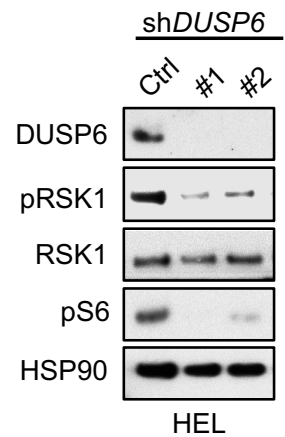

PI3K-AKT signaling pathway

Complement and coagulation

ECM-receptor interaction

Protein processing in endoplasmic reticulum

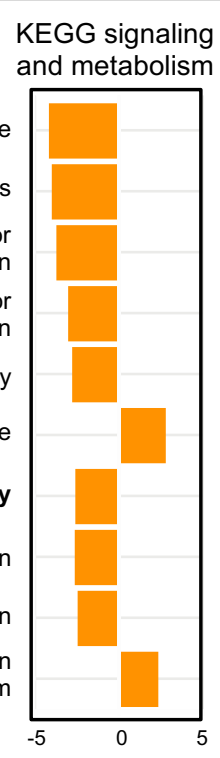

Mean log2 fold-change

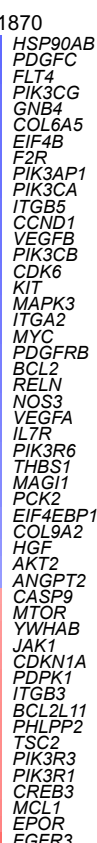


A

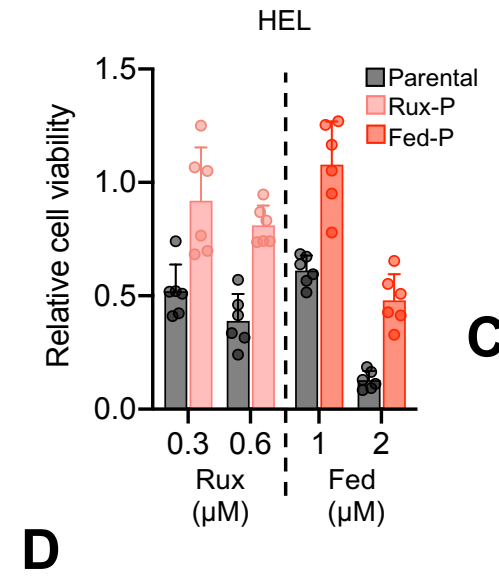

HEL

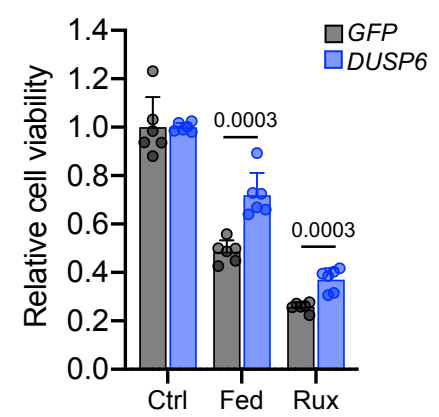

E

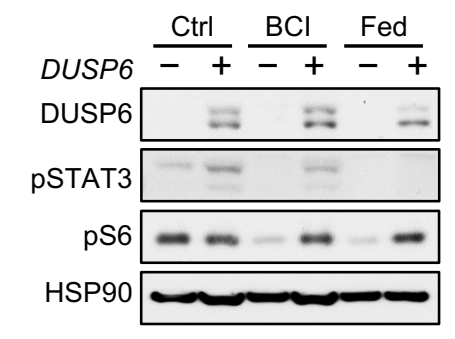

HEL
B

mRNA fold change

relative to HEL parental

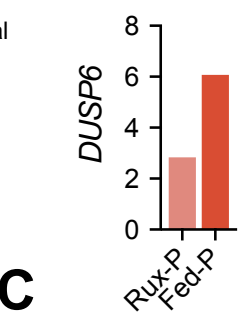

DUSP6 target genes UP
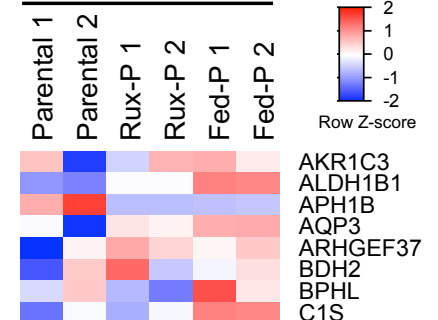

DDH2

$\mathrm{C} 1 \mathrm{~S}$

CD24

CNTNAP1

CTTNBP2N DUSP4

ENPP5 4 A

HERC5

HERC6

HMOX1

ID3

KCIM1

KLRC2

MALAT1

MAPK11

MDK

MUC20
OFD1

PARP9

PSMB9

PYCARD

RCN3

RNASE1 1

SECTM1

SLC2A10

SMIM14

SNED 1

ST6GALNAC2

TAPBPL

THEMIS2

TMCO

HEL
F

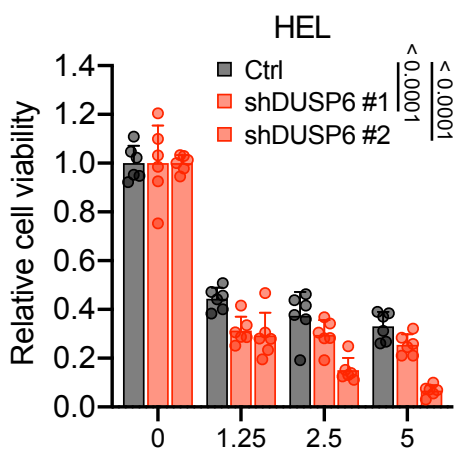

G

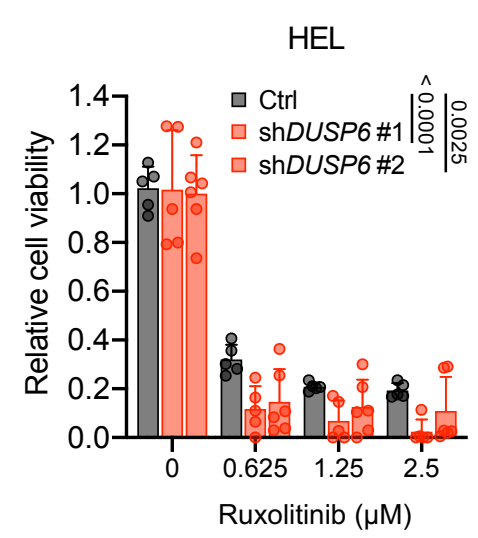

H

Figure 5
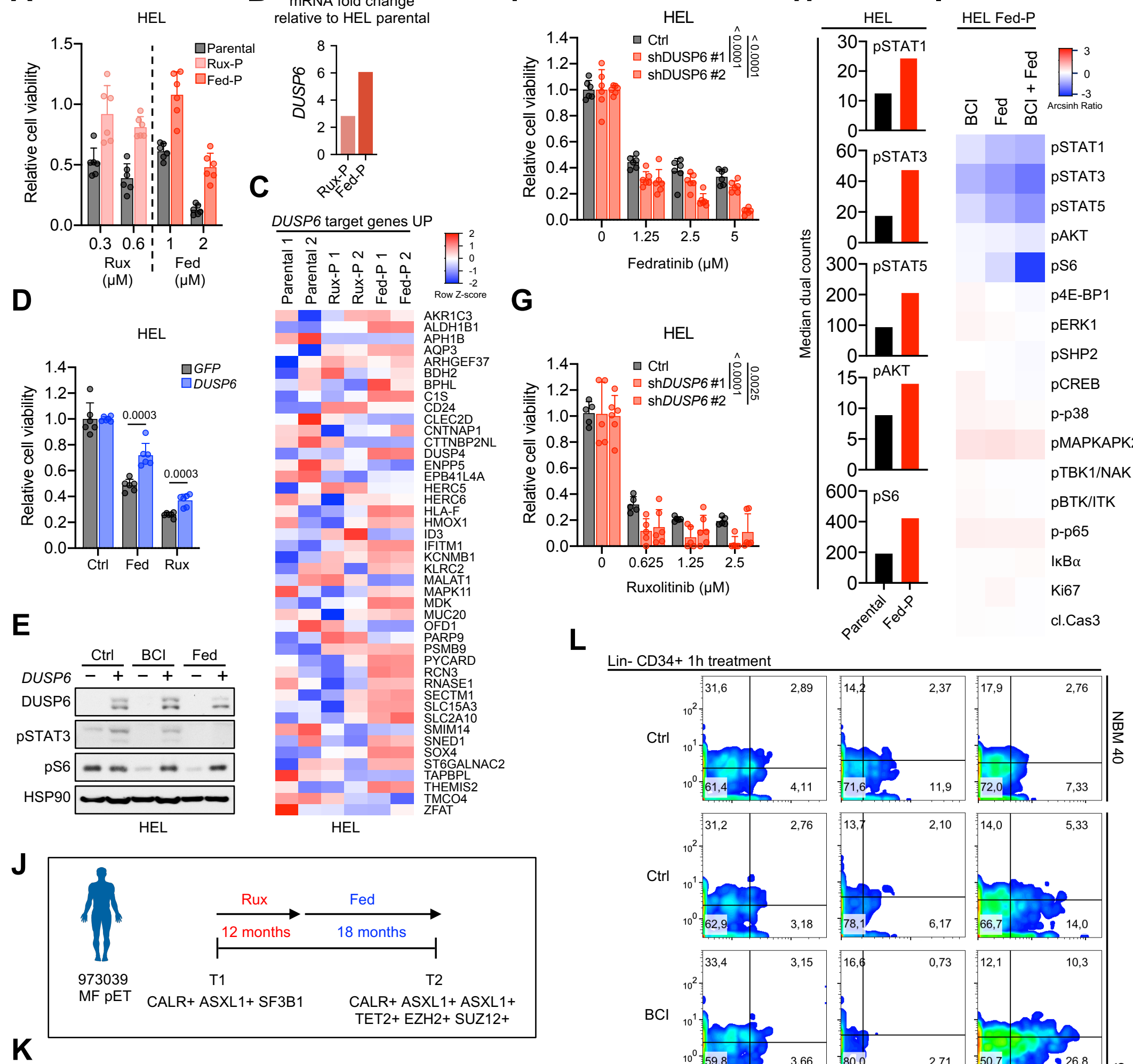

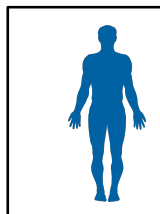

973039

MF pET

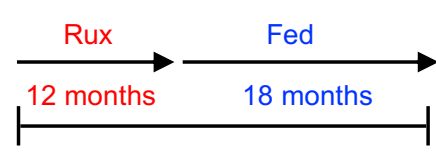

T1

T2

ALR+ ASXL1+ ASXL1+ TET2+ EZH2+ SUZ12+
K

CD34+ colony assay Erythroid $\square$ Myeloid

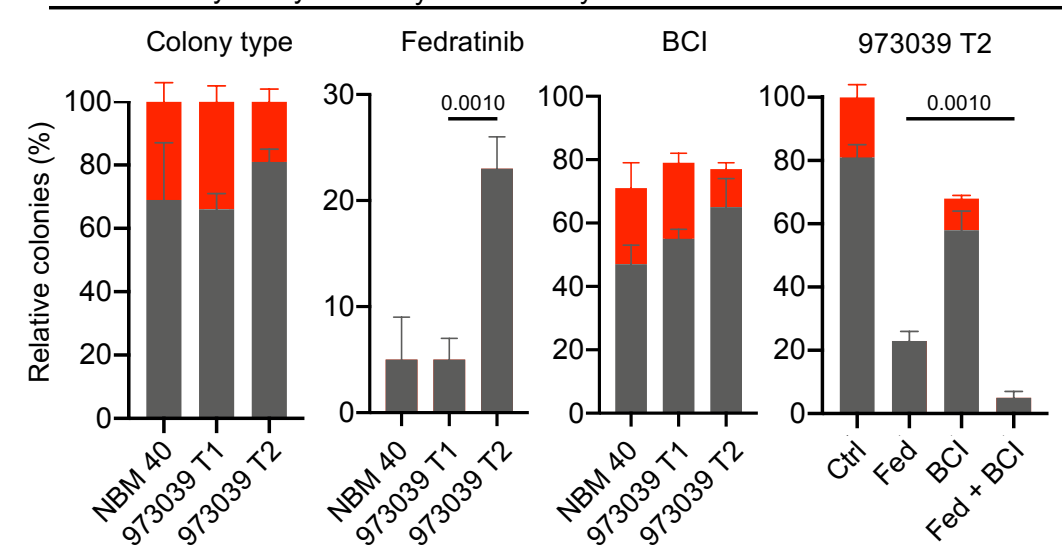

Fedratinib $(\mu \mathrm{M})$

HEL

L
Lin- CD34+ $1 \mathrm{~h}$ treatment
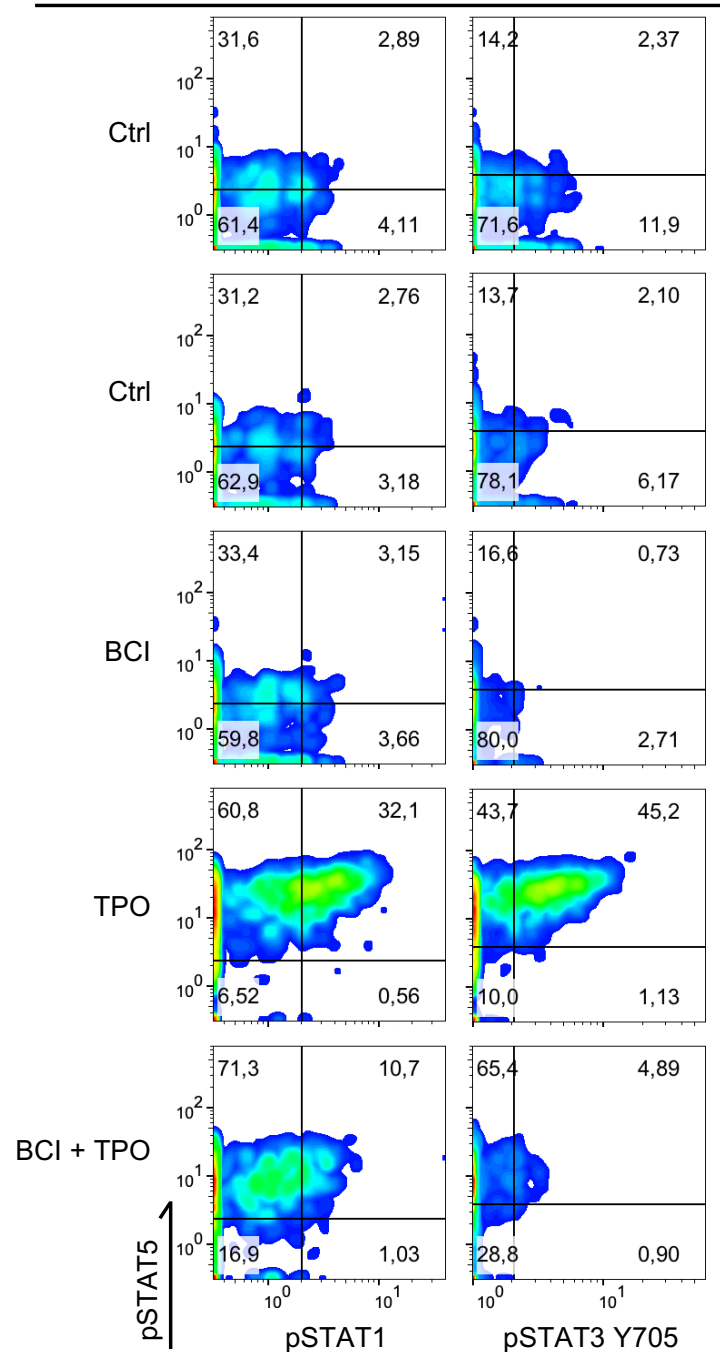
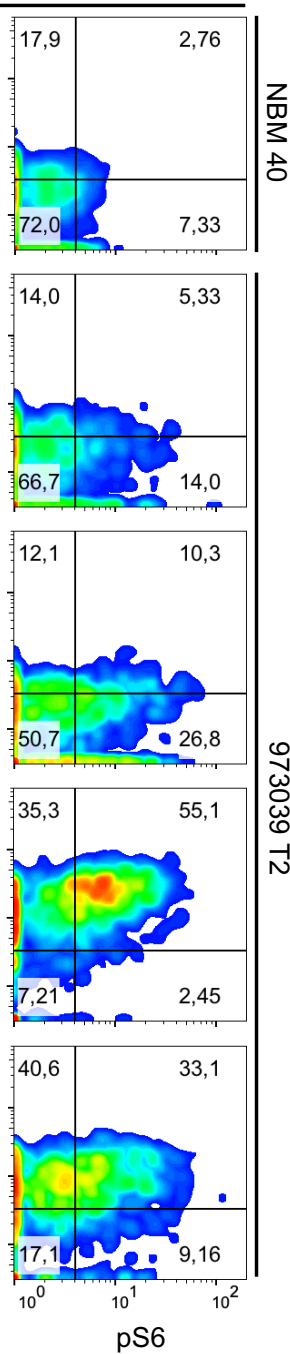
Figure 6
A
B

Jak2 V617F

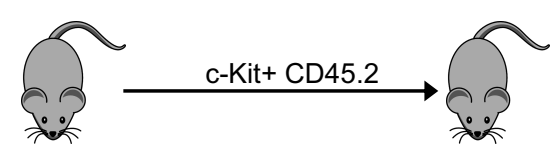

Jak2 V617F polycythemia vera model
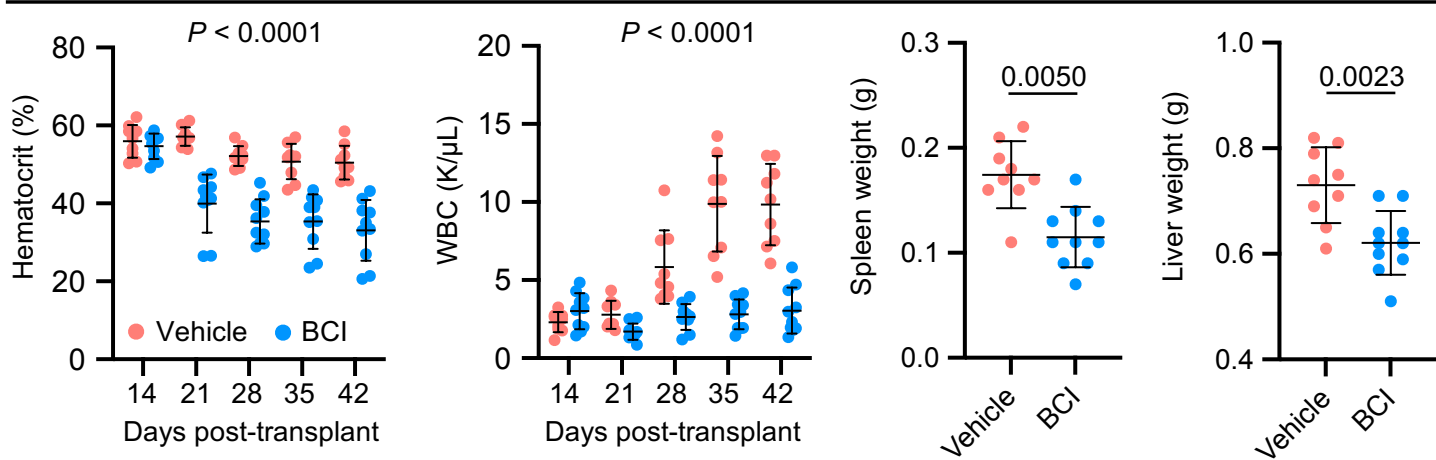

C

D

MPL W515L myelofibrosis model
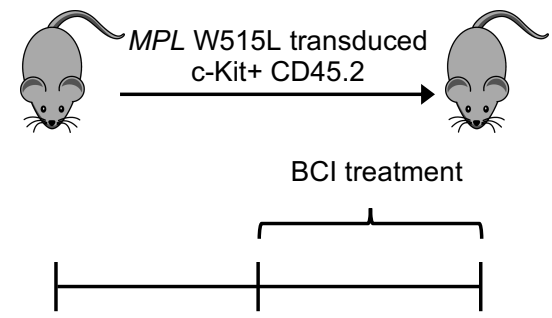

0

Days post-transplant

E

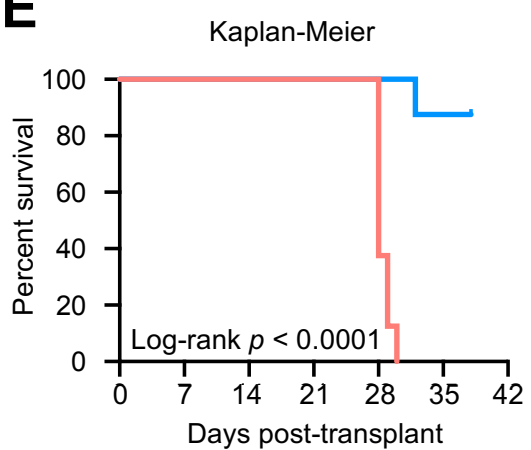

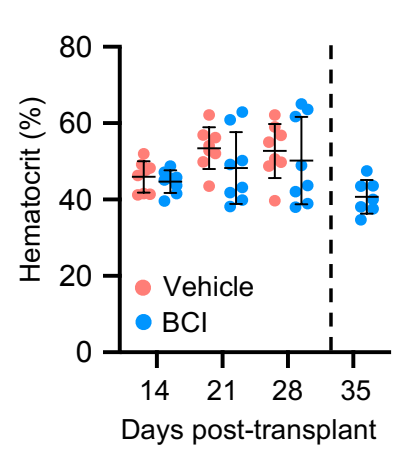
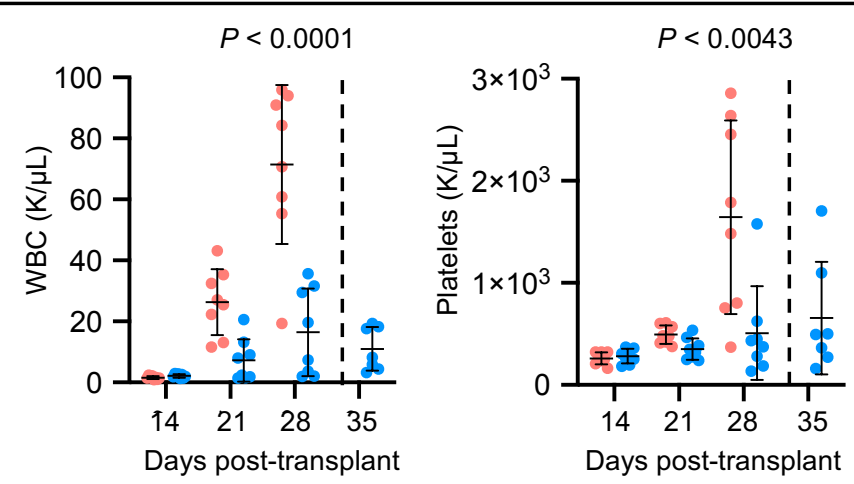

F
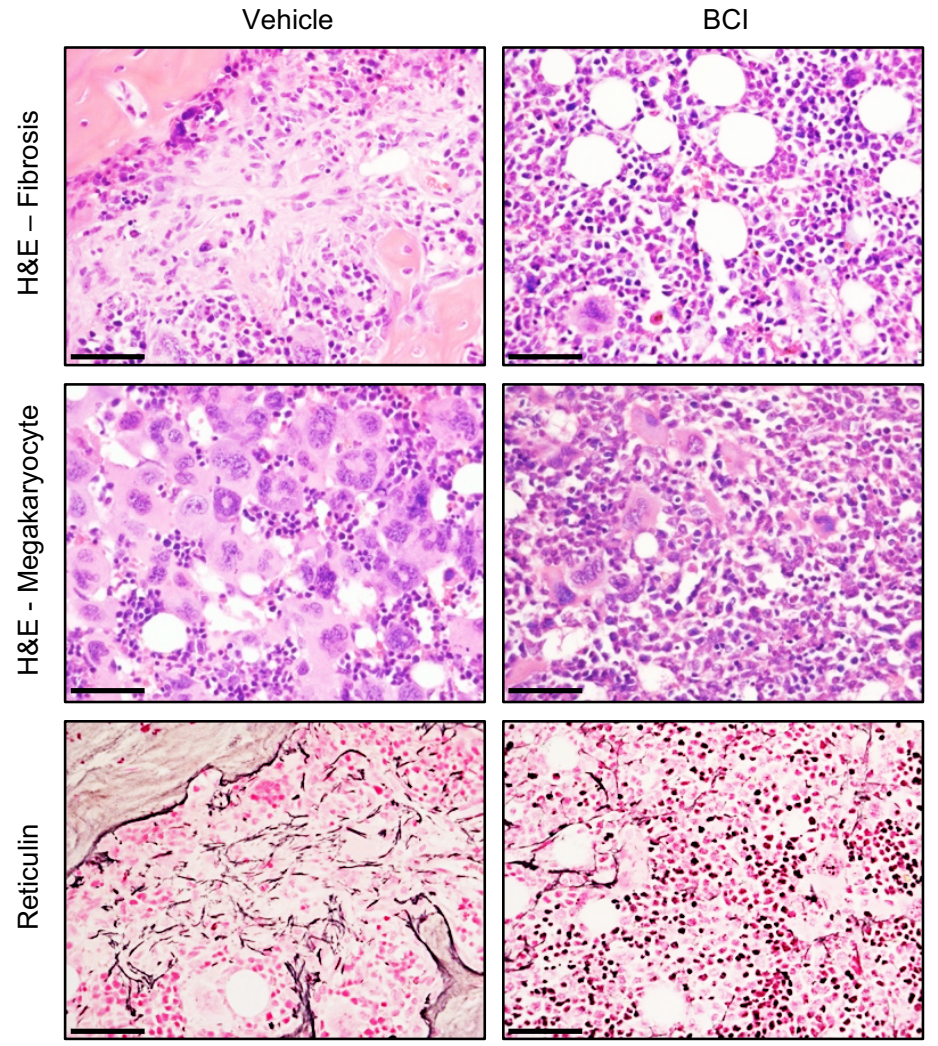
Figure 7

A

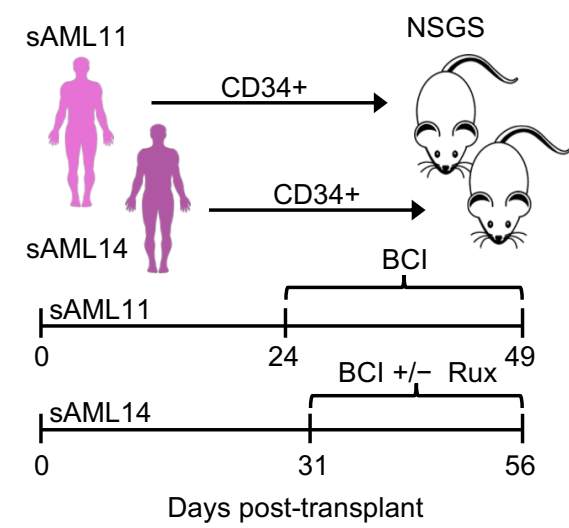

B

sAML11 CD34+ PDX

C

sAML14 CD34+ PDX
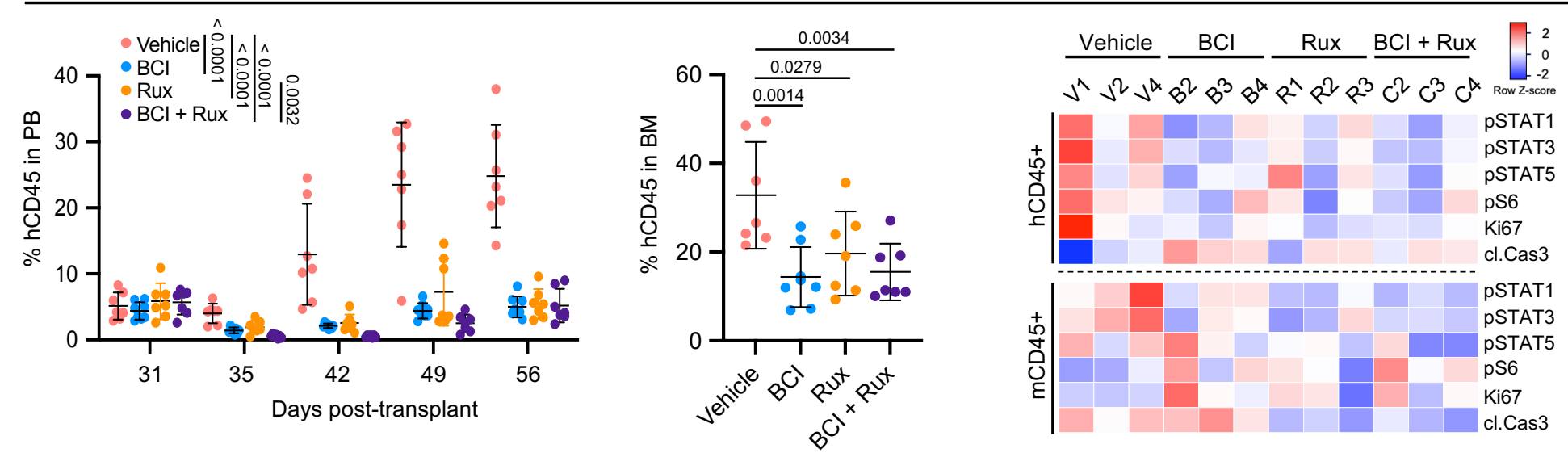